\title{
SUPER AND COMBINATORIAL HARMONIC RESPONSE OF FLEXIBLE ELASTIC CABLES WITH SMALL SAG
}

\author{
S. R. K. Nielsen and P. H. KirkegaArd \\ Department of Civil Engineering, Aalborg University, Sohngaardsholmsvej 57, 9000 Aalborg, Denmark. \\ E-mail:i6phk@civil.auc.dk
}

(Received 23 February 2001, and in final form 27 July 2001)

\begin{abstract}
The paper deals with the analysis of cables in stayed bridges and TV-towers, where the excitation is caused by harmonically varying in-plane motions of the upper support point with the amplitude $U$. Such cables are characterized by a sag-to-chord-length ratio below $0 \cdot 02$, which means that the lowest circular eigenfrequencies for in-plane and out-of-plane eigenvibrations, $\omega_{1}$ and $\omega_{2}$, are closely separated. The dynamic analysis is performed by a two-degree-of-freedom modal decomposition in the lowest in-plane and out-of-plane eigenmodes. Modal parameters are evaluated based on the eigenmodes for the parabolic approximation to the equilibrium suspension. Superharmonic components of the order $n$, supported by the parametric terms of the excitation and the non-linear coupling terms, are registered in the response for circular frequency $\omega \simeq \omega_{1} / n$. At moderate $U$, the cable response takes place entirely in the static equilibrium plane. At larger amplitudes the in-plane response becomes unstable and a coupled whirling superharmonic component occurs. In the paper a first order perturbation solution to the superharmonic response is performed based on the averaging method. For $\omega \simeq(m / n) \omega_{1}, m<n$, the geometrical non-linear restoring forces gives rise to a substantial combinatorial harmonic component with the circular frequency $(n / m) \omega$. Both entirely in-plane and coupled in-plane and out-of-plane responses occur. Based on an initial frequency analysis of the response, an analytical model for these vibrations is formulated with emphasis on superharmonics of the order $n=3$ and combinatorial harmonics of the order $(n, m)=(3,2)$. All analytical solutions have been verified by direct numerical integration of the modal equations of motion.
\end{abstract}

(C) 2002 Elsevier Science Ltd.

\section{INTRODUCTION}

Linear free and forced oscillations of elastic cables with a small sag-to-chord-length ratio based on the parabolic equilibrium approximation were first developed by Irvine [1] and Irvine and Caughey [2]. Based on a single-degree-of-freedom model for the in-plane vibrations of the cable, Hagedorn and Shafer [3] extended the linear theory by considering the effect of quadratic and cubic non-linearities on eigenfrequencies. An extension of this research was given by Luongo et al. [4, 5] and Rega et al. [6], who showed that the dynamic behaviour becomes either hardening or softening depending on the relative contributions on the quadratic and cubic non-linearities determined by the cable parameter and the amplitude of oscillation. Coupled in-plane and out-of-plane eigenvibrations were investigated in reference [7] showing a beating type exchange of energy between the two modes. In-plane harmonic response caused by an external harmonically varying load per unit length of the cable was analyzed by Benedettini and Rega [8] using a single-degree-of-freedom model. Forced response with coupled in-plane and out-of-plane response has been analyzed in a number of studies based on two-degree-of-freedom models. 
Al-Noury and Ali [9] considered the harmonic response due to a harmonically varying load per unit length of the cable, using a two-degree-of-freedom Galerkin approach with sine functions used as shape functions, identical to the eigenmodes of the taut wire. The same problem was dealt with by Rao and Iyengar [10], who used the eigenmodes of the parabolic equilibrium approximation as a functional basis at the reduction to a two-degree-of-freedom system. Additionally, an incremental Lyapunov stability analysis of the obtained harmonic solutions was performed. The use of the parabolic suspension is restricted to relatively small sag-to-chord-length ratios. Coupled non-linear in-plane and out-of-plane eigenvibrations of cables with large sag were analyzed numerically by Takahashi and Konishi [11] based on a multi-degree-of-freedom Galerkin approach with sine functions as the functional basis. The primarily external excitation of cables used in stay bridges and TV-towers is caused by the motion of the support points of the cable rather than by external distributed dynamic loads. The effect of forced support motions has been considered by Perkins [12], who obtained analytical solutions based on a first order perturbation analysis of a two-degree-of-freedom model for coupled in-plane and out-of-plane response using the eigenmodes of the parabolic approximation to the static equilibrium suspension as a functional basis. The emphasis was placed on cables with relatively large sag-to-chord-length ratios to analyze a so-called 2:1 resonance, which indicates that an in-plane primary harmonic resonance in combination with a subharmonic resonance of the order $\frac{1}{2}$ occurs in the out-of-plane modal co-ordinate. Lee and Perkins [13] extended the work to include second order perturbations and multiple internal resonances. Still, the focus was on 2:1 resonance, whereas the excitation was changed to a harmonically varying load per unit length acting in the static equilibrium plane. The same load was considered by Lee and Perkins [14] with emphasis on combined harmonic resonance in the in-plane and out-of-plane modal co-ordinates. It was demonstrated that the stable response to the in-plane excitation at a certain vibration level was a whirling motion involving a phase lag of $\pi / 2$ between the modal co-ordinates. Recently, Benedettini et al. [15] have investigated cable response phenomena of an elastic suspended cable subjected to both in-plane harmonically varying external loads per unit length and in-plane support-point motions. The focus was on a four-degree-of-freedom model containing two in-plane and two out-of-plane components, and both 2:1 resonance and harmonic resonance were studied based on a first order perturbation analysis of the discretized cable equations, i.e., 1:1 internal resonance and subharmonic external resonance of the order $\frac{1}{2}$ were considered. Possible modes of steady state motions of the cable were identified and stability was verified by a linearized stability analysis. An experimental detection of these different types of planar and non-planar regular motions are presented in references [16, 17]. Asymptotic models for the complicated non-linear three-dimensional responses of an elastic suspended shallow cable to a harmonic excitation were derived in reference [18] by using the method of multiple scales directly to the governing partial-differential equations and a four-degrees-of-freedom discretized model respectively.

In civil engineering, cables with small sag are used as support of cable-stayed bridges and TV-towers, where the support-point motions may be in any direction relative to the static equilibrium state. Such cables are characterized by a sag-to-chord-length ratio below $0 \cdot 02$, which means that the lowest circular eigenfrequencies for in-plane and out-of-plane eigenvibrations, $\omega_{1}$ and $\omega_{2}$, are closely separated. Moreover, the small sag-to-chord-length ratio means that the circular frequency of the support point motion, displaying the lowest circular eigenfrequency of the bridge or the TV-tower, in most cases is well below the lowest circular eigenfrequencies $\omega_{1} \simeq \omega_{2}$. Under these conditions, the cable is prone to superharmonic response at critical excitation frequencies. Previously, in-plane superharmonic response of the orders $n=2$ and 3 has been considered by Benedettini and 
Rega [19], using the plane model given in reference [8]. No analyses of superharmonic response caused by the endpoint motions have been reported in the literature. In particular, the analysis of the coupled in-plane and out-of-plane superharmonic response is absent. Furthermore, combinatorial harmonic response due to support motion is completely ignored. These subjects are the concern of the present paper.

The superharmonic response has been analyzed based on a two-degree-of-freedom model, derived from the equations of motion for flexible linear elastic cables under excitation of forced support point motions in combination with a modal expansion in the two lowest eigenmodes of the parabolic approximation to the static equilibrium suspension state. The method of analysis is based on an initial co-ordinate transformation from physical to van der Pol co-ordinates followed by an averaging over the excitation, assuming weak non-linearities and corresponding slowly varying phases and amplitudes. It is demonstrated that the in-plane superharmonic response to the in-plane excitation remains stable up to a certain critical vibration level, at which a coupled whirling motion is generated by bifurcation from the in-plane response. In the case of circular excitation frequencies $\omega \simeq(m / n) \omega_{1}$, it is demonstrated that the geometrical non-linear couplings result in substantial combinatorial harmonic components. Although significant amplifications are still present, combinatorial response levels are somewhat smaller than the comparable superharmonic vibrations. Only combinatorial harmonics of the order $(n, m)=(3,2)$ have been analyzed. As for the superharmonic response, both an in-plane and a coupled in-plane and out-of-plane combinatorial mode of oscillation may coexist at a certain circular excitation frequency.

\section{THEORY OF ELASTIC CABLES}

Figure 1 shows the cable in the static equilibrium state, where the support points of the cable are fixed and where the cable is in equilibrium with the external static load per unit length of the equilibrium state, $p_{i}^{(0)}\left(s_{0}\right)$. $s_{0}$ is an arch parameter measured from one end of the cable. The total length of the cable in the static equilibrium state is $L_{0}$. The deformed state of the cable is caused by time-varying forced displacements $U_{i}^{(l)}(t)$ and $U_{i}^{(u)}(t)$ of the lower and the upper support points of the cable. The vectors $U_{i}^{(l)}(t)$ and $U_{i}^{(u)}(t)$ are measured relative to the fixed Cartesian $\left(x_{1}, x_{2}, x_{3}\right)$-co-ordinate system as is the case with all subsequently defined vectors and tensors. Typically, $U_{i}^{(l)}(t)$ represents the vertical displacement of the bridge deck, whereas $U_{i}^{(u)}(t)$ is the horizontal motion of the pylon or



Figure 1. Static equilibrium state and deformed state of cable. 
TV-tower at the upper support point. The summation convention over dummy indices has been extensively used. The cable is assumed to be linearly elastic at all deformations from the static equilibrium state. Additionally, the cable is perfectly flexible against bending deformations.

Consider a cable element $\mathrm{d} s_{0}$ in the static equilibrium state at the position $x_{i}^{(0)}(s, t)=x_{i}^{(0)}\left(s_{0}\right)$. During the deformation the cable element is stretched, rotated and translated into the cable element $\mathrm{d} s$ of the deformed state at the position $x_{i}(s, t)=x_{i}\left(s_{0}, t\right)$. The referential and displaced positions of the cable are given by the relation

$$
x_{i}\left(s_{0}, t\right)=x_{i}^{(0)}\left(s_{0}\right)+u_{i}\left(s_{0}, t\right),
$$

where $u_{i}\left(s_{0}, t\right)$ is the incremental displacement of the cable element. The cable forces in the referential and in the deformed states are denoted as $F_{i}^{(0)}\left(s_{0}\right)$ and $F_{i}\left(s_{0}, t\right)$ respectively. These are related as follows:

$$
F_{i}\left(s_{0}, t\right)=F_{i}^{(0)}\left(s_{0}\right)+\Delta F_{i}\left(s_{0}, t\right) .
$$

The equations of motion for the considered cable element are

$$
\frac{\partial F_{i}\left(s_{0}, t\right)}{\partial s_{0}}-\mu_{0} \frac{\partial^{2} u_{i}}{\partial t^{2}}=p_{i}^{(0)}\left(s_{0}\right) \Rightarrow \frac{\partial\left(\Delta F_{i}\left(s_{0}, t\right)\right)}{\partial s_{0}}-\mu_{0} \frac{\partial^{2} u_{i}}{\partial t^{2}}=0,
$$

where $\mu_{0}$ is the mass per unit length of the static equilibrium state. Because the cable has been assumed to be perfectly flexible the cable forces in both configurations are tangential, i.e.,

$$
F_{i}^{(0)}\left(s_{0}\right)=F^{(0)}\left(s_{0}\right) t_{i}^{(0)}\left(s_{0}\right), \quad F_{i}\left(s_{0}, t\right)=F\left(s_{0}, t\right) t_{i}\left(s_{0}, t\right),
$$

where $t_{i}^{(0)}\left(s_{0}\right)$ and $t_{i}\left(s_{0}, t\right)$ are the tangential unit vectors and $F^{(0)}\left(s_{0}\right)$ and $F\left(s_{0}, t\right)$ are the moduli of the corresponding cable forces. The tangential unit vectors in the two configurations are given as

$$
t_{i}^{(0)}\left(s_{0}\right)=\frac{\partial x_{i}^{(0)}\left(s_{0}\right)}{\partial s_{0}}, \quad t_{i}\left(s_{0}, t\right)=\frac{\partial x_{i}\left(s_{0}, t\right)}{\partial s} .
$$

From equation (1) it follows that

$$
t_{i}=\left(t_{i}^{(0)}+\frac{\partial u_{i}}{\partial s_{0}}\right) \frac{\mathrm{d} s_{0}}{\mathrm{~d} s} \Rightarrow\left(\frac{\mathrm{d} s}{\mathrm{~d} s_{0}}\right)^{2}=1+2 \frac{\partial u_{i}}{\partial s_{0}} t_{i}^{(0)}+\frac{\partial u_{i}}{\partial s_{0}} \frac{\partial u_{i}}{\partial s_{0}},
$$

leading to the following expression for the axial strain:

$$
\varepsilon=\frac{\mathrm{d} s-\mathrm{d} s_{0}}{\mathrm{~d} s_{0}}=-1+\sqrt{1+2 \frac{\partial u_{i}}{\partial s_{0}} t_{i}^{(0)}+\frac{\partial u_{i}}{\partial s_{0}} \frac{\partial u_{i}}{\partial s_{0}}} .
$$

Assuming linear elasticity at all deformations from the static equilibrium state the increment of the cable force is given as

$$
\Delta F=F-F^{(0)}=A E \varepsilon,
$$


where $A$ is the cross-sectional area in the referential state and $E$ is the elasticity modulus. The vectorial force increment follows from equation (4)

$$
\begin{aligned}
\Delta F_{i}\left(s_{0}, t\right) & =F t_{i}-F^{(0)} t_{i}^{(0)}=\Delta F t_{i}+F^{(0)}\left(t_{i}-t_{i}^{(0)}\right) \\
& =A E \frac{\varepsilon}{1+\varepsilon}\left(t_{i}^{(0)}+\frac{\partial u_{i}}{\partial s_{0}}\right)+F^{(0)}\left(\frac{1}{1+\varepsilon}\left(t_{i}^{(0)}+\frac{\partial u_{i}}{\partial s_{0}}\right)-t_{i}^{(0)}\right) \\
& =A_{i j} \frac{\partial u_{j}}{\partial s_{0}}+B_{i}\left(\frac{\partial u_{k}}{\partial s_{0}}\right),
\end{aligned}
$$

where the linear and non-linear parts of the vectorial force increment are defined as

$$
\begin{gathered}
A_{i j}=\left(A E-F^{(0)}\right) t_{i}^{(0)} t_{j}^{(0)}+F^{(0)} \delta_{i j} \\
B_{i}\left(\frac{\partial u_{k}}{\partial s_{0}}\right)=\left(A E-F^{(0)}\right)\left(\frac{\varepsilon}{1+\varepsilon} t_{i}^{(0)}-\frac{\partial u_{j}}{\partial s_{0}} t_{j}^{(0)} t_{i}^{(0)}+\frac{\varepsilon}{1+\varepsilon} \frac{\partial u_{i}}{\partial s_{0}}\right) .
\end{gathered}
$$

Eqution (11) is valid even for finite axial strains. However, in reality, it may be assumed that $\varepsilon \ll 1$, i.e., with an error of magnitude $\varepsilon^{2}$ one has $\varepsilon /(1+\varepsilon) \simeq \varepsilon$.

Furthermore, the axial strain in the referential state $F^{(0)} / A E$ may be assumed to be small compared to 1. Equations (10) and (11) may then be written as

$$
\begin{gathered}
A_{i j}=A E t_{i}^{(0)} t_{j}^{(0)}+F^{(0)} \delta_{i j} \\
B_{i}\left(\frac{\partial u_{k}}{\partial s_{0}}\right)=\frac{1}{2} A E\left(\frac{\partial u_{j}}{\partial s_{0}} \frac{\partial u_{j}}{\partial s_{0}} t_{i}^{(0)}+2 \frac{\partial u_{j}}{\partial s_{0}} t_{j}^{(0)} \frac{\partial u_{i}}{\partial s_{0}}+\frac{\partial u_{j}}{\partial s_{0}} \frac{\partial u_{j}}{\partial s_{0}} \frac{\partial u_{i}}{\partial s_{0}}\right)
\end{gathered}
$$

The right-hand side of equation (13) specifies the consistent quadratic and cubic terms of the cable force increment on the condition that the dynamic and static axial strains remain sufficiently small.

\section{MODAL DECOMPOSITION}

The incremental displacement field is written in the form

$$
u_{i}\left(s_{0}, t\right)=U_{i}^{(l)}(t)\left(1-\frac{s_{0}}{L_{0}}\right)+U_{i}^{(u)}(t) \frac{s_{0}}{L_{0}}+\sum_{\alpha=1}^{\infty} \Phi_{i}^{(\alpha)}\left(s_{0}\right) q_{\alpha}(t),
$$

where $q_{\alpha}(t)$ are the modal co-ordinates and $\Phi_{i}^{(\alpha)}\left(s_{0}\right)$ are the linear undamped eigenmodes of the cable. In the following, the summation convention is retained for lower case Latin indices but is abandoned for Greek indices. The eigenmodes are obtained from the following linear eigenvalue problem (cf., equations $(3,9)$ ):

$$
\frac{\mathrm{d}}{\mathrm{d} s_{0}}\left(A_{i j} \frac{\mathrm{d}}{\mathrm{d} s_{0}} \Phi_{j}^{(\alpha)}\right)+\mu_{0} \omega_{\alpha}^{2} \Phi_{i}^{(\alpha)}=0, \quad \Phi_{i}^{(\alpha)}(0)=\Phi_{i}^{(\alpha)}\left(L_{0}\right)=0,
$$


where $A_{i j}\left(s_{0}\right)$ is given by equation (12), and $\omega_{\alpha}$ are the undamped circular eigenfrequencies. Since $A_{i j}=A_{j i}$, the eigenvalue problem is self-adjoint and the eigenmodes fulfil the orthogonality properties

$$
\begin{gathered}
\int_{0}^{L_{0}} \mu_{0} \Phi_{i}^{(\alpha)} \Phi_{i}^{(\beta)} \mathrm{d} s_{0}= \begin{cases}0, & \alpha \neq \beta, \\
M_{\alpha}, & \alpha=\beta,\end{cases} \\
\int_{0}^{L_{0}} \frac{\mathrm{d} \Phi_{i}^{(\alpha)}}{\mathrm{d} s_{0}} A_{i j}\left(s_{0}\right) \frac{\mathrm{d} \Phi_{j}^{(\beta)}}{\mathrm{d} s_{0}} \mathrm{~d} s_{0}= \begin{cases}0, & \alpha \neq \beta, \\
\omega_{\alpha}^{2} M_{\alpha}, & \alpha=\beta,\end{cases}
\end{gathered}
$$

where $M_{\alpha}$ is the modal mass

$$
M_{\alpha}=\int_{0}^{L_{0}} \mu_{0} \Phi_{i}^{(\alpha)} \Phi_{i}^{(\alpha)} \mathrm{d} s_{0} .
$$

Insertion of equation (14) into the incremental equation of motion (3), with $\Delta F_{i}$ given by equations (9), (12), (13), provides the following ordinary differential equations for the modal co-ordinates $q_{\alpha}(t)$ :

$$
\ddot{q}_{\alpha}+2 \zeta_{\alpha} \omega_{\alpha} \dot{q}_{\alpha}+\omega_{\alpha}^{2} q_{\alpha}+b_{\alpha}\left(q_{\gamma}, t\right)=f_{\alpha}(t), \quad \alpha=1,2, \ldots
$$

where

$$
\begin{gathered}
f_{\alpha}(t)=-\frac{\ddot{U}_{i}^{(l)}(t)}{M_{\alpha}} \int_{0}^{L_{0}} \mu_{0} \Phi_{i}^{(\alpha)}\left(s_{0}\right)\left(1-\frac{s_{0}}{L_{0}}\right) \mathrm{d} s_{0}-\frac{\ddot{U}_{i}^{(u)}(t)}{M_{\alpha}} \int_{0}^{L_{0}} \mu_{0} \Phi_{i}^{(\alpha)}\left(s_{0}\right) \frac{s_{0}}{L_{0}} \mathrm{~d} s_{0} \\
-\frac{e_{i}(t)}{M_{\alpha}} \int_{0}^{L_{0}} A_{i j}\left(s_{0}\right) \frac{\mathrm{d} \Phi_{j}^{(\alpha)}}{\mathrm{d} s_{0}} \mathrm{~d} s_{0}, \\
b_{\alpha}\left(q_{\gamma}, t\right)=\frac{1}{M_{\alpha}} \int_{0}^{L_{0}} \frac{\mathrm{d} \Phi_{i}^{(\alpha)}}{\mathrm{d} s_{0}} B_{i}\left(e_{k}(t)+\sum_{\beta=1}^{\infty} \frac{\mathrm{d} \Phi_{k}^{(\beta)}}{\mathrm{d} s_{0}} q_{\beta}\right) \mathrm{d} s_{0}, \\
e_{i}(t)=\frac{1}{L_{0}}\left(U_{i}^{(u)}(t)-U_{i}^{(l)}(t)\right) .
\end{gathered}
$$

As seen, a linear viscous damping term with the modal damping ratios $\zeta_{\alpha}$ has been included in equation (19). $b_{\alpha}\left(q_{\gamma}, t\right)$ determines the coupling and the level of parametric excitation in the modal co-ordinate differential equations. Insertion of equation (13) into equation (21) provides the following expansion for the modal coupling terms:

$$
\begin{aligned}
b_{\alpha}\left(q_{\gamma}, t\right)= & a_{j k}^{(\alpha)} e_{j} e_{k}+e_{j} \sum_{\beta} c_{j}^{(\alpha \beta)} q_{\beta}+\sum_{\beta, \gamma} d^{(\alpha \beta \gamma)} q_{\beta} q_{\gamma} \\
& +e_{j} e_{k} \sum_{\beta} C_{j k}^{(\alpha \beta)} q_{\beta}+e_{j} \sum_{\beta, \gamma} D_{j}^{(\alpha \beta \gamma)} q_{\beta} q_{\gamma}+\sum_{\beta, \gamma, \delta} G^{(\alpha \beta \gamma \delta)} q_{\beta} q_{\gamma} q_{\delta} .
\end{aligned}
$$


The tensor components entering equation (23) become

$$
\begin{gathered}
a_{j k}^{(\alpha)}=\frac{A E}{2 M_{\alpha}} \int_{0}^{L_{0}} \frac{\mathrm{d} \Phi_{i}^{(\alpha)}}{\mathrm{d} s_{0}}\left(t_{i}^{(0)} \delta_{j k}+2 \delta_{i j} t_{k}^{(0)}\right) \mathrm{d} s_{0} \\
c_{j}^{(\alpha \beta)}=\frac{A E}{2 M^{\alpha}} \int_{0}^{L_{0}} 2 \frac{\mathrm{d} \Phi_{i}^{(\alpha)}}{\mathrm{d} s_{0}}\left(t_{i}^{(0)} \frac{\mathrm{d} \Phi_{j}^{(\beta)}}{\mathrm{d} s_{0}}+\delta_{i j} t_{k}^{(0)} \frac{\mathrm{d} \Phi_{k}^{(\beta)}}{\mathrm{d} s_{0}}+\frac{\mathrm{d} \Phi_{i}^{(\beta)}}{\mathrm{d} s_{0}} t_{j}^{(0)}\right) \mathrm{d} s_{0}, \\
d^{(\alpha \beta \gamma)}=\frac{A E}{2 M_{\alpha}} \int_{0}^{L_{0}} \frac{\mathrm{d} \Phi_{i}^{(\alpha)}}{\mathrm{d} s_{0}}\left(t_{i}^{(0)} \frac{\mathrm{d} \Phi_{j}^{(\beta)}}{\mathrm{d} s_{0}} \frac{\mathrm{d} \Phi_{j}^{(\gamma)}}{\mathrm{d} s_{0}}+2 \frac{\mathrm{d} \Phi_{i}^{(\beta)}}{\mathrm{d} s_{0}} \frac{\mathrm{d} \Phi_{j}^{(\gamma)}}{\mathrm{d} s_{0}} t_{j}^{(0)}\right) \mathrm{d} s_{0} \\
C_{j k}^{(\alpha \beta)}=\frac{A E}{2 M_{\alpha}} \int_{0}^{L_{0}} \frac{\mathrm{d} \Phi_{i}^{(\alpha)}}{\mathrm{d} s_{0}}\left(2 \delta_{i j} \frac{\mathrm{d} \Phi_{k}^{(\beta)}}{\mathrm{d} s_{0}}+\frac{\mathrm{d} \Phi_{i}^{(\beta)}}{\mathrm{d} s_{0}} \delta_{j k}\right) \mathrm{d} s_{0} \\
D_{j}^{(\alpha \beta \gamma)}=\frac{A E}{2 M_{\alpha}} \int_{0}^{L_{0}} \frac{\mathrm{d} \Phi_{i}^{(\alpha)}}{\mathrm{d} s_{0}}\left(2 \frac{\mathrm{d} \Phi_{i}^{(\beta)}}{\mathrm{d} s_{0}} \frac{\mathrm{d} \Phi_{j}^{(\gamma)}}{\mathrm{d} s_{0}}+\delta_{i j} \frac{\mathrm{d} \Phi_{k}^{(\beta)}}{\mathrm{d} s_{0}} \frac{\mathrm{d} \Phi_{k}^{(\gamma)}}{\mathrm{d} s_{0}}\right) \mathrm{d} s_{0} \\
G^{(\alpha \beta \gamma \delta)}=\frac{A E}{2 M_{\alpha}} \int_{0}^{L_{0}} \frac{\mathrm{d} \Phi_{i}^{(\alpha)}}{\mathrm{d} s_{0}} \frac{\mathrm{d} \Phi_{i}^{(\beta)}}{\mathrm{d} s_{0}} \frac{\mathrm{d} \Phi_{j}^{(\gamma)}}{\mathrm{d} s_{0}} \frac{\mathrm{d} \Phi_{j}^{(\delta)}}{\mathrm{d} s_{0}} \mathrm{~d} s_{0} .
\end{gathered}
$$

Lower case and upper case tensors are caused by quadratic and cubic non-linearities respectively. The important terms in equation (23) as well as their physical implications will be identified below. The present investigation concentrates on the analysis of the non-linear couplings between the two lowest modes of the cable. These couplings are only of significance when both modes are subjected to resonance, i.e., $\omega \simeq \omega_{1} \simeq \omega_{2}$, so the corresponding modal coordinates are relatively large. Then the higher modal co-ordinates $q_{3}(t), q_{4}(t), \ldots$ will all be insignificant compared to $q_{1}(t)$ and $q_{2}(t)$. Hence, the modal expansions in equations (14) and (23) may be truncated after the first two modal co-ordinates. The theory presumes simultaneous resonance in the first and second mode because of which the lowest circular frequencies $\omega_{1}$ and $\omega_{2}$ should differ at most by $20 \%$, which limits the sag-to-chord-length ratio to $f / L_{c} \leqslant 0 \cdot 02$.

The static equilibrium is assumed to be caused by gravity. Then the equilibrium state is placed in a vertical plane through the two support points. In such cases, dynamic analysis based on the parabolic approximation to the static equilibrium suspension may be used for sag-to-chord-length ratios up to $f / L_{c} \simeq 0.08$ [1]. With the Cartesian co-ordinate placed as shown in Figure 2 the parabolic suspension is given as

$$
\begin{gathered}
y\left(x_{1}\right)=4 f \frac{x_{1}}{L_{c}}\left(1-\frac{x_{1}}{L_{c}}\right) \Rightarrow t_{i}^{(0)}\left(x_{1}\right)=\left[\begin{array}{c}
1 \\
\left.\frac{4 f}{L_{c}}\left(1-2 \frac{x_{1}}{L_{c}}\right)\right] \frac{\mathrm{d} x_{1}}{\mathrm{~d} s_{0}}, \quad \frac{\mathrm{d} x_{1}}{\mathrm{~d} s_{0}} \simeq 1-8 \frac{f^{2}}{L_{c}^{2}}\left(1-2 \frac{x_{1}}{L_{c}}\right)^{2}, \\
0
\end{array}\right] \\
\frac{f}{L_{c}}=\frac{1}{8} \frac{\mu_{0} g \cos \theta L_{c}}{F_{c}},
\end{gathered}
$$




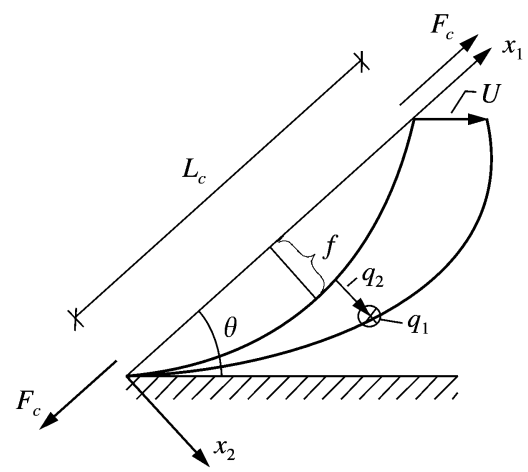

Figure 2. Definition of parameters and Cartesian co-ordinate system.

where $f$ is the sag, $L_{c}$ is the chord length, $\theta$ is the inclination of the chord length and $g$ is the acceleration of gravity. $F_{c}$ is the average of the component along the chord of the variable static cable force $F_{i}^{(0)}\left(s_{0}\right)$.

Based on the parabolic approximation, the lowest eigenmodes of the eigenvibrations in-plane and lateral to the static equilibrium state become [1]

$$
\begin{gathered}
\boldsymbol{\Phi}_{i}^{(1)}\left(x_{1}\right)=\left[\begin{array}{c}
0 \\
0 \\
\sin \left(\pi \frac{x_{1}}{L_{c}}\right)
\end{array}\right], \quad \omega_{1}=\frac{\pi}{L_{c}} \sqrt{\frac{F_{c}}{\mu_{0}}}, \\
\boldsymbol{\Phi}_{i}^{(2)}\left(x_{1}\right)=\left[\begin{array}{c}
0 \\
\Phi^{(2)}\left(x_{1}\right) \\
0
\end{array}\right], \quad \omega_{2}=\frac{\Omega_{2}}{L_{c}} \sqrt{\frac{F_{c}}{\mu_{0}}},
\end{gathered}
$$

where

$$
\Phi^{(2)}\left(x_{1}\right)=\frac{\left[\cos \left(\left(\Omega_{2} / 2\right)\left(1-2 x_{1} / L_{c}\right)\right)-\cos \Omega_{2} / 2\right.}{\left(1-\cos \Omega_{2} / 2\right)} .
$$

The eigenmodes have been normalized to 1 at the midpoint of the cable. Then $q_{1}(t)$ and $q_{2}(t)$ may be interpreted as the amplitudes of the displacement of the midpoint. The non-dimensional eigenfrequency $\Omega_{2}$ is the lowest positive solution to the transcendental equation

$$
\tan \frac{\Omega}{2}=\frac{\Omega}{2}-\frac{4}{\lambda^{2}}\left(\frac{\Omega}{2}\right)^{3}, \quad \lambda^{2}=\frac{A E}{F_{c}} \frac{64\left(f / L_{c}\right)^{2}}{1+(8 / 3)\left(f / L_{c}\right)^{2}} .
$$

The corresponding modal masses are

$$
M_{\alpha}=\mu_{0} \int_{0}^{L_{c}} \Phi_{\alpha}^{2}\left(x_{1}\right) \mathrm{d} x= \begin{cases}\frac{\mu_{0} L_{c}}{2}, & \alpha=1 \\ \frac{\mu_{0} L_{c}}{2} \frac{\left(2+\cos \Omega_{2}-\left(2 / \Omega_{2}\right) \sin \Omega_{2}\right)}{\left(1-\cos \Omega_{2} / 2\right)^{2}}, & \alpha=2 .\end{cases}
$$


Given the eigenmodes (27), modal load (20) and the tensor components (24) may be explicitly evaluated. At the evaluation, only terms of the zeroth and first order in $f / L_{c}$ are retained. Consistently, one may use the approximation $\mathrm{d} x_{1} / \mathrm{d} s_{0} \simeq 1$ in equation (25), equivalent to replacing the cable length $L_{0}$ by the chord length $L_{c}$. In the following the lower support-point of the cable is assumed to be fixed, whereas the upper support-point is performing horizontal harmonic motions in the static equilibrium plane with the circular frequency $\omega$ as given by (see Figure 2)

$$
U_{i}^{(u)}(t)=U\left[\begin{array}{c}
\cos \theta \\
\sin \theta \\
0
\end{array}\right] \cos (\omega t)
$$

From equation (31) the following results may be derived for the non-dimensional support-point displacement $e_{i}(t)$ and the modal loads:

$$
\begin{array}{cl}
e_{i}(t)=E_{i} \cos (\omega t), \quad \mathbf{E}_{i}=\frac{U}{L_{c}}\left[\begin{array}{c}
\cos \theta \\
\sin \theta \\
0
\end{array}\right], & \alpha=1, \\
f_{\alpha}(t)=F_{\alpha} \cos (\omega t), \quad F_{\alpha}=U \begin{cases}0, & \\
a\left(\omega^{2} \sin \theta-16 \frac{f}{L_{c}} \frac{A E}{\mu_{0} L_{c}^{2}} \cos \theta\right), & \alpha=2,\end{cases}
\end{array}
$$

where the coefficient $a$ is given in equation (77) in the appendix. Endpoint displacements with a component in the out-of-plane direction result in a modal load of the magnitude $F_{1}=U a \omega^{2}$, which is small in the case of superharmonic response, where $\omega=\omega_{1} / n$. For this reason, the applied in-plane excitation is considered as adequate for a qualitative description of the superharmonic response even for endpoint displacements with components both in the in-plane and in the out-of-plane directions. The second term within the bracket in the expression for $F_{2}$ is caused by the elongation of the chord, which implies a negative quasistatic response of $q_{2}$. The stationary harmonic response of the modal co-ordinates for the linear version of system (19) with $b_{\alpha}\left(q_{1}, q_{2}, t\right)=0$ becomes

$$
\begin{gathered}
q_{1,0}(t)=0, \quad q_{2,0}(t)=B_{0} \cos \left(\omega t+\Psi_{0}\right), \\
B_{0}=\frac{F_{2}}{\sqrt{\left(\omega_{2}^{2}-\omega^{2}\right)^{2}+4 \zeta_{2}^{2} \omega_{2}^{2} \omega^{2}}}, \quad \tan \Psi_{0}=\frac{2 \zeta_{2} \omega_{2} \omega}{\omega^{2}-\omega_{2}^{2}} .
\end{gathered}
$$

The linear harmonic solution may be considered the zeroth order solution in a perturbation analysis. However, not all the terms are equally important. A closer inspection based on the linear solution (34) reveals that only the second and the last term in equation (23) turn out to be important, which results in the following expression for the coupling terms (see Appendix A):

$$
b_{\alpha}\left(q_{1}, q_{2}, t\right) \simeq \frac{A E}{\mu_{0} L_{c}^{2}} \begin{cases}\left(e_{1} c_{1}+\frac{1}{L_{c}^{2}}\left(G_{1} q_{1}^{2}+G_{2} q_{2}^{2}\right)\right) q_{1}, & \alpha=1, \\ \left(e_{1} c_{2}+\frac{1}{L_{c}^{2}}\left(G_{3} q_{1}^{2}+G_{4} q_{2}^{2}\right)\right) q_{2}, & \alpha=2,\end{cases}
$$


$c_{1}, c_{2}, G_{2}, G_{3}, G_{4}$ are all given as a function of $\Omega_{2}$ in equation (82). For a taut wire $G_{1}=G_{2}=G_{3}=G_{4}=\frac{3}{8} \pi^{4}$. Although the difference is small the coefficients for a cable with small sag are ordered as $G_{3}>G_{4}>G_{1}>G_{2}$. As can be seen, only the component along the chord line $e_{1}(t)=\left(U / L_{c}\right) \cos \theta \cos (\omega t)$ of the non-dimensional support-point displacement causes parametric excitations of significance in the equations of motion.

\section{SUPERHARMONIC RESPONSE OF TWO-DEGREE-OF-FREEDOM MODEL}

The two-degree-of-freedom model in equations (19) and (35) applies for circular excitation frequency $\omega<\omega_{1}$, where higher modes are insignificantly excited. For $\omega \simeq \omega_{1} / n$, $n=2,3, \ldots$ a significant additional superharmonic component of the order $n$ with the circular frequency $n \omega$ appears in the response in addition to the linear harmonic response in equation (34). The superharmonic components are maintained through resonance excitation from the parametric and geometrical non-linear coupling terms in equation (35). The superharmonic response may take place entirely in the equilibrium plane and as coupled vibrations of the in-plane and out-of-plane modal coordinates. For these vibrations the response is assumed tobe in the form

$$
q_{\alpha}(t)=q_{\alpha, 0}(t)+Q_{\alpha, n}(t) \cos \Phi_{\alpha, n}(t), \quad \dot{q}_{\alpha}(t)=\dot{q}_{\alpha, 0}(t)-n \omega Q_{\alpha, n}(t) \sin \Phi_{\alpha, n}(t)
$$

where

$$
\Phi_{\alpha, n}(t)=n \omega t+\Psi_{\alpha, n}(t)
$$

If equation (36) is a valid representation both for $q_{\alpha}(t)$ and $\dot{q}_{\alpha}(t)$, the derivative of $q_{\alpha}(t)$ must give the second relation. This provides the so-called consistence condition

$$
\dot{Q}_{\alpha, n} \cos \Phi_{\alpha, n}-Q_{\alpha, n} \dot{\Psi}_{\alpha, n} \sin \Phi_{\alpha, n}=0
$$

Assuming $Q_{\alpha, n}(t) \geqslant 0$ and $\left.\left.\omega t+\Psi_{\alpha, n}(t) \in\right] 0,2 \pi\right]$, equation (36) is merely a one-to-one mapping between the dynamic state variables $q_{\alpha}(t)$ and $\dot{q}_{\alpha}(t)$ and the so-called van der Pol state variables $Q_{\alpha, n}(t)$ and $\Psi_{\alpha, n}(t)$. Upon insertion of equation (36) into equation (19), $q_{\alpha, 0}(t)$ will counterbalance the excitation $f_{\alpha}(t)=F_{\alpha} \cos (\omega t)$ when inserted into the linear part. Use of the consistence condition then provides the following differential equation for the van der Pol state variables:

$$
\begin{aligned}
n \omega \dot{Q}_{\alpha, n}= & -n^{2} \omega^{2} Q_{\alpha, n} \cos \Phi_{\alpha, n} \sin \Phi_{\alpha, n}-2 \zeta_{\alpha} \omega_{\alpha} n \omega Q_{\alpha, n} \sin ^{2} \Phi_{\alpha, n} \\
& +\omega_{\alpha}^{2} Q_{\alpha, n} \cos \Phi_{\alpha, n} \sin \Phi_{\alpha, n}+b_{\alpha}(t) \sin \Phi_{\alpha, n}, \\
n \omega Q_{\alpha, n} \dot{\Psi}_{\alpha, n}= & -n^{2} \omega^{2} Q_{\alpha, n} \cos ^{2} \Phi_{\alpha, n}-2 \zeta_{\alpha} \omega_{\alpha} n \omega Q_{\alpha, n} \sin \Phi_{\alpha, n} \cos \Phi_{\alpha, n} \\
& +\omega_{\alpha}^{2} Q_{\alpha, n} \cos ^{2} \Phi_{\alpha, n}+b_{\alpha}(t) \cos \Phi_{\alpha, n} .
\end{aligned}
$$

In case of superharmonic response, $Q_{\alpha, n}(t)$ and $\Psi_{\alpha, n}(t)$ are constant in time and $q_{\alpha}(t)$ is a period function with time with the period $T=2 \pi / \omega$. For the time being, the van der Pol variables are merely considered as a slowly varying function with time. The so-called averaging method, identical to the first order Krylov-Bogoliubov-Mitropolsky perturbation solution [20], presumes that the long-term development of the van der Pol 
variables can be determined from the differential equations obtained, when the right-hand sides of equation (39) are replaced by the time average over the excitation period $T$, and with $Q_{\alpha, n}(t)$ and $\Psi_{\alpha, n}(t)$ kept constant during the time averaging. This leads to the following averaged differential equations:

$$
\begin{gathered}
n \dot{\omega} Q_{\alpha, n}=-\zeta_{\alpha} \omega_{\alpha} n \omega Q_{\alpha, n}+\frac{1}{T} \int_{0}^{T} b_{\alpha}(t) \sin \Phi_{\alpha, n} \mathrm{~d} t \\
n \omega Q_{\alpha, n} \dot{\Psi}_{\alpha}=\frac{1}{2}\left(\omega_{\alpha}^{2}-n^{2} \omega^{2}\right) Q_{\alpha, n}+\frac{1}{T} \int_{0}^{T} b_{\alpha}(t) \cos \Phi_{\alpha, n} \mathrm{~d} t .
\end{gathered}
$$

$q_{\alpha}(t)$ as given by equation (36) is inserted into equation (40) and the time averaging is evaluated. The following differential equations may be derived for the superharmonic component of the order $n=2,3, \ldots$.

$$
\begin{aligned}
2 n \omega \dot{A}= & -2 \zeta_{1} \omega_{1} n \omega A+\gamma_{2} A B^{2} \Phi, \\
2 n \omega \dot{B}= & -2 \zeta_{2} \omega_{2} n \omega B-\gamma_{3} A^{2} B \sin 2 \Phi-P_{n} \sin \left(\alpha_{n}-\Psi_{2}\right), \\
2 n \omega A \dot{\Psi}_{1}= & \left(\omega_{1}^{2}-n^{2} \omega^{2}+\gamma_{2} 2 B_{0}^{2}\right) A+\gamma_{1} A^{3}+\gamma_{2}(2+\cos 2 \Phi) A B^{2}, \\
2 n \omega B \dot{\Psi}_{2}= & \left(\omega_{2}^{2}-n^{2} \omega^{2}+\gamma_{4} 2 B_{0}^{2}\right) B+\gamma_{4} B^{3}+\gamma_{3}(2+\cos \Phi) A^{2} B \\
& +P_{n} \cos \left(\alpha_{n}-\Psi_{2}\right),
\end{aligned}
$$

where the abbreviated notion $\left[A, B, \Psi_{1}, \Psi_{2}\right]$ has been used for $\left[Q_{1, n}, Q_{2, n}, \Psi_{1, n}, \Psi_{2, n}\right]$. Furthermore, the following symbols have been introduced:

$$
\begin{gathered}
\Phi(t)=\Psi_{1}(t)-\Psi_{2}(t), \quad \beta_{1}=\frac{1}{2} \frac{A E}{\mu_{0} L_{c}^{2}} E_{1} c_{1}, \quad \beta_{2}=\frac{1}{2} \frac{A E}{\mu_{0} L_{c}^{2}} E_{1} c_{2}, \\
\gamma_{1}=\frac{3}{4} \frac{A E}{\mu_{0} L_{c}^{4}} G_{1}, \quad \gamma_{2}=\frac{1}{4} \frac{A E}{\mu_{0} L_{c}^{4}} G_{2}, \quad \gamma_{3}=\frac{1}{4} \frac{A E}{\mu_{0} L_{c}^{4}} G_{3}, \quad \gamma_{4}=\frac{3}{4} \frac{A E}{\mu_{0} L_{c}^{4}} G_{4}, \\
P_{n}=\left\{\begin{array}{ll}
\beta_{2} B_{0}, & n=2, \\
\frac{1}{3} \gamma_{4} B_{0}^{3}, & n=3, \\
0, & n=4,5, \ldots,
\end{array} \quad \alpha_{n}= \begin{cases}\Psi_{0}, & n=2, \\
3 \Psi_{0}, & n=3, \\
0, & n=4,5, \ldots\end{cases} \right.
\end{gathered}
$$

As seen from equation (45), at the considered first order perturbation level, the superharmonic response of the order $n=2$ is entirely caused by the parametric excitation via the term $e_{1} c_{2} q_{2}$ (revealed by the factor $\beta_{2}$ ), whereas the superharmonic response of the order $n=3$ is generated by the zeroth order solution through the geometrical non-linear couplings. Equations (41) are autonomous differential equations for the determination of the superharmonic components of the response, which can all be recast into the following vectorial format:

$$
\dot{\mathbf{x}}(t)=\mathbf{f}(\mathbf{x}(t)), \quad \mathbf{x}^{\mathrm{T}}(t)=\left[A(t), B(t), \Psi_{1}(t), \Psi_{2}(t)\right]
$$

Superharmonic components are harmonic functions with time, i.e., $\mathbf{x}(t) \equiv$ $\mathbf{x}^{(0)}=\left[A^{(0)}, B^{(0)}, \Psi_{1}^{(0)}, \Psi_{2}^{(0)}\right]$ is a constant vector. This means that the right-hand side of 
equation (46) is identically equal to zero, i.e., possible superharmonic response components are determined by the equilibrium points (singular points) of equation (46). Hence,

$$
\mathbf{f}\left(\mathbf{x}^{(0)}\right)=\mathbf{0}
$$

The existence of possible solutions to equation (47) does not guarantee that the corresponding superharmonic components are stable. However, stability of the said harmonic components can be studied from the stability of the equilibrium points determined by equation (47). The following perturbation is introduced:

$$
\mathbf{x}(t)=\mathbf{x}^{(0)}+\Delta \mathbf{x}(t)
$$

Introduction of equation (48) into equation (46) and the use of equation (47) leads to the following first order variational equation for the increment $\Delta \mathbf{x}(t)$, valid in the vicinity of $\mathbf{x}^{(0)}$

$$
\Delta \dot{\mathbf{x}}(\mathrm{t})=\mathbf{A} \Delta \mathbf{x}(\mathrm{t}), \quad \mathbf{A}=\frac{\partial \mathbf{f}\left(\mathbf{x}^{(0)}\right)}{\partial \mathbf{x}}
$$

Infinitesimal Lyapunov instability of the equilibrium points can then be studied from the stability properties of the variational equations (49). The equilibrium point, and hence the corresponding superharmonic components, are asymptotically stable if all eigenvalues of the gradient matrix A have negative real part. The equilibrium state and the underlying superharmonic motion are unstable if at least one eigenvalue has positive real part, Meirovitch [17]. Possible superharmonic components of the order $n$ are determined from the equilibrium points of equation (41) leading to the following non-linear algebraic equations:

$$
\begin{aligned}
& -2 n \zeta_{1} \omega_{1} \omega A+\gamma_{2} A B^{2} \operatorname{Sin} 2 \Phi=0, \\
& -2 n \zeta_{2} \omega_{2} \omega B-\gamma_{3} A^{2} B \operatorname{Sin} 2 \Phi=P_{n} \sin \left(\alpha_{n}-\Psi_{2}\right), \\
& \left(\omega_{1}^{2}-n^{2} \omega^{2}+2 \gamma_{2} B_{0}^{2}\right) A+\gamma_{1} A^{3}+\gamma_{2}(2+\cos 2 \Phi) A B^{2}=0, \\
& \left(\omega_{2}^{2}-n^{2} \omega^{2}+2 \gamma_{4} B_{0}^{2}\right) B+\gamma_{4} B^{3}+\gamma_{3}(2+\cos 2 \Phi) A^{2} B 1=-P_{n} \cos \left(\alpha_{n}-\Psi_{2}\right) .
\end{aligned}
$$

At first, the in-plane response is determined, i.e., it is assumed that $A=0$. Then the first and third equations in equation (50) are automatically fulfilled. The second and fourth equations provide the following relations for $B=Q_{2, n}$ and $\Psi_{2}=\Psi_{2, n}$ :

$\left(4 n^{2} \zeta_{2}^{2} \omega_{2}^{2} \omega^{2}+\left(\omega_{2}^{2}-n^{2} \omega^{2}+2 \gamma_{4} B_{0}^{2}\right)^{2}\right) B^{2}+2 \gamma_{4}\left(\omega_{2}^{2}-n^{2} \omega^{2}+2 \gamma_{4} B_{0}^{2}\right) B^{4}+\gamma_{4}^{2} B^{6}=P_{n}^{2}$,

$\tan \left(\alpha_{n}-\Psi_{2}\right)=2 \zeta_{2} \omega_{2} n \omega \omega_{2}^{2}-n^{2} \omega^{2}+\gamma_{4} 2 B_{0}^{2}+\gamma_{4} B^{2}$

Equation (51) is obtained by squaring and summing the second and the fourth equations of equations (50), and equation (52) is obtained by division of the second equation by the fourth equation. Equation (51) has up to three positive real solutions for $B^{2}$, each representing a possible superharmonic motion. For each of the possible solutions the corresponding phase is determined from equation (52). Next, motions with $A \neq 0$ are 
analyzed. Then, the first equation in equation (50) can only be fulfilled for

$$
\operatorname{Sin} 2 \Phi=\frac{2 n \zeta_{1} \omega_{1} \omega}{\gamma_{2} B^{2}} \Rightarrow \cos 2 \Phi= \pm \sqrt{1-\frac{4 n^{2} \zeta_{1}^{2} \omega_{1}^{2} \omega^{2}}{\gamma_{2}^{2} B^{4}}} .
$$

To achieve a qualitative overview over possible solutions the undamped case $\zeta_{1}=0$ is considered. Then

$$
\sin 2 \Phi=0 \Rightarrow \Psi_{1}-\Psi_{2}=\left\{\begin{array}{ll}
0, & \pi \\
\frac{\pi}{2}, & \frac{3 \pi}{2}
\end{array} \Rightarrow \operatorname{Cos} 2 \Phi=\left\{\begin{array}{r}
1 \\
-1
\end{array}\right.\right.
$$

For $\Psi_{1}-\Psi_{2}=0, \pi$ the two superharmonic co-ordinates move in phase or counterphase, corresponding to a phase motion taking place in a plane with an inclined angle to the equilibrium plane determined by the amplitudes $A$ and $B$. For $\Psi_{1}-\Psi_{2}=\pi / 2,3 \pi / 2$ the superharmonics move in a whirling ellipsoidal motion with the semiaxes $A$ and $B$. In the actual damped case the same two types of solutions persist, where + and - signs in equation (53) refer to phase and whirling motions respectively. The third equation in equation (50) provides

$$
A^{2}=-\frac{1}{\gamma_{1}}\left(\omega_{1}^{2}-n^{2} \omega^{2}+2 \gamma_{2} B_{0}^{2}\right)-\frac{\gamma_{2}}{\gamma_{1}}(2+\cos 2 \Phi) B^{2} .
$$

$A^{2}$ is eliminated in the second and fourth equation of equation (50). After squaring and summing these equations, the following non-linear equation in $B^{2}$ is obtained for the determination of the amplitude of possible superharmonic motions

$$
a B^{2}+b B^{4}+c B^{6}=P_{n}^{2}
$$

where

$$
\begin{aligned}
a= & 4 n^{2} \zeta_{2}^{2} \omega_{2}^{2} \omega^{2}+\left(\omega_{2}^{2}-n^{2} \omega^{2}+2 \gamma_{4} B_{0}^{2}\right)^{2}-2 \frac{\gamma_{3}}{\gamma_{1}} \zeta_{2} \omega_{2} n \omega\left(\omega_{1}^{2}-n^{2} \omega^{2}+2 \gamma_{2} B_{0}^{2}\right) \sin 2 \Phi \\
& -2 \frac{\gamma_{3}}{\gamma_{1}}(2+\cos 2 \Phi)\left(\omega_{1}^{2}-n^{2} \omega^{2}+2 \gamma_{2} B_{0}^{2}\right)\left(\omega_{2}^{2}-n^{2} \omega^{2}+2 \gamma_{4} B_{0}^{2}\right) \\
& +\frac{\gamma_{3}^{2}}{\gamma_{1}^{2}}(5+4 \cos 2 \Phi)\left(\omega_{1}^{2}-n^{2} \omega^{2}+2 \gamma_{2} B_{0}^{2}\right)^{2} \\
b= & 2 \gamma_{4}\left(\omega_{2}^{2}-n^{2} \omega^{2}+2 \gamma_{4} B_{0}^{2}\right)-2 \frac{\gamma_{2} \gamma_{3}}{\gamma_{1}}(2+\cos 2 \Phi)^{2}\left(\omega_{2}^{2}-n^{2} \omega^{2}+2 \gamma_{4} B_{0}^{2}\right) \\
& -2 \frac{\gamma_{3} \gamma_{4}}{\gamma_{1}}(2+\cos 2 \Phi)\left(\omega_{1}^{2}-n^{2} \omega^{2}+2 \gamma_{2} B_{0}^{2}\right)-2 \frac{\gamma_{2} \gamma_{3}}{\gamma_{1}} \zeta_{2} \omega_{2} n \omega(2+\cos 2 \Phi) \sin 2 \Phi \\
& +2 \frac{\gamma_{2} \gamma_{3}^{2}}{\gamma_{1}^{2}}(2+\cos 2 \Phi)(5+4 \cos 2 \Phi)\left(\omega_{1}^{2}-n^{2} \omega^{2}+2 \gamma_{2} B_{0}^{2}\right), \\
c= & \gamma_{4}^{2}-\frac{\gamma_{2} \gamma_{3}}{\gamma_{1}}(2+\cos 2 \Phi)^{2}\left(2 \gamma_{4}-\frac{\gamma_{2} \gamma_{3}}{\gamma_{1}}(5+4 \cos 2 \Phi)\right) .
\end{aligned}
$$


For light damping, the coefficients $a, b$ and $c$ depend weakly on $B^{2}$ through $\sin 2 \Phi$ and $\cos 2 \Phi$ as follows from equation (53). Hence, equation (56) is not a cubic equation, although up to three positive solutions for $B^{2}$ may still exist. Solutions must be obtained by cyclic iteration using the undamped solutions $\sin 2 \Phi=0 \wedge \cos 2 \Phi= \pm 1$ as start values. Phase motions and whirling motions are obtained by iterations with $\cos 2 \Phi=+$ $\sqrt{1-4 n^{2} \zeta_{1}^{2} \omega_{1}^{2} \omega^{2} /\left(\gamma_{2}^{2} B^{4}\right)}$ and $\cos 2 \Phi=-\sqrt{1-4 n^{2} \zeta_{1}^{2} \omega_{1}^{2} \omega^{2} /\left(\gamma_{2}^{2} B^{4}\right)}$ respectively. The corresponding solution for $A^{2}$ follows from equation (55). Both $A^{2}$ and $B^{2}$ must be positive for possible superharmonic solutions. With $A$ and $B$ determined by equations (55) and (56), division of the second equation by the fourth equation provides the following solution for the phase $\Psi_{2}=\Psi_{2, n}$ :

$$
\tan \left(\alpha_{n}-\Psi_{2}\right)=\frac{2 \zeta_{2} \omega_{2} n \omega+\gamma_{3} A^{2} \sin 2 \Phi}{\omega_{2}^{2}-n^{2} \omega^{2}+\gamma_{4} 2 B_{0}^{2}+\gamma_{4} B^{2}+\gamma_{3}(2+\cos 2 \Phi) A^{2}}
$$

Finally, the phase $\Psi_{1}=\Psi_{1, n}$ is determined from the proper solutions to equation (53) for $\Phi=\Psi_{1}-\Psi_{2}$, which correspond to the assumed sign of $\sin 2 \Phi$ and $\cos 2 \Phi$. The motion with $A \neq 0$ is bifurcating from the plane motion with $A=0$ at a critical point $C=\left(\omega_{c}, B_{c}\right)$, at which equation (55) is fulfilled with $A=0$. Then it follows that

$$
\omega_{c}^{2}=\frac{1}{n^{2}}\left(\omega_{1}^{2}+2 \gamma_{2} B_{0}^{2}+\gamma_{2}(2+\cos 2 \Phi) B_{c}^{2}\right)
$$

If $\omega=T \omega_{c}$ is eliminated in equation (57) as well as in the phase condition $\Phi=-\sqrt{1-4 n^{2} \zeta_{1}^{2} \omega_{1}^{2} \omega^{2} /\left(\gamma_{2}^{2} B^{4}\right)}$, the amplitude $B_{c}$ may be obtained from the resulting equation. Whirling superharmonic motions are present even for a taut-wire case. However, since $\omega_{1}=\omega_{2}$ in this case, both the bifurcation amplitude $B_{c}$ and the critical circular frequency $\omega_{c}$ will be different in this case.

\section{COMBINATORIAL HARMONIC RESPONSE}

Whereas superharmonic response as indicated by equation (36) is primarily dominated only by two frequencies, the situation becomes more complicated in the case of superharmonic response of the order $(n, m)=(3,2)$. To reveal the relevant harmonic components, a Fourier analysis of modal co-ordinates $q_{1}(t)$ and $q_{2}(t)$, obtained by numerical integration of equations (19) and (35) with the initial values of $q_{1}(0)=q_{2}(0)=10 \mathrm{~m}, \dot{q}_{1}(0)=\dot{q}_{2}(0)=0$ has been performed as shown in Figure $3(\mathrm{a})$ and 3 (b). The lowest circular eigenfrequency of the considered cable, with mechanical and geometrical data as given in the numerical example below, is $\omega_{1}=3 \cdot 1476 / \mathrm{s}$, and the amplitude of the excitation is $U=0.5 \mathrm{~m}$. The excitation frequency is $\omega=2 \cdot 1 / \mathrm{s}$, which is pretty close to $\frac{2}{3} \omega_{1}$. Time has been normalized with respect to the excitation period $T=2 \pi / \omega$ in the time series. $\Omega$ and $\omega$ in the plots of the Fourier transform indicate the running circular frequency and the circular excitation frequency respectively. As seen in Figure 3(a), a subharmonic component with the circular frequency $\frac{1}{2} \omega$ and a substantial combinatorial harmonic component with the circular frequency $\frac{3}{2} \omega$ is present in the out-of-plane modal component $q_{1}(t)$. The in-plane component $q_{2}(t)$ in Figure $3(\mathrm{~b})$ is dominated by a harmonic component with the circular frequency $\omega$ and a superharmonic component with the frequency $2 \omega$ in addition to a static drift. Figure 4 shows the 


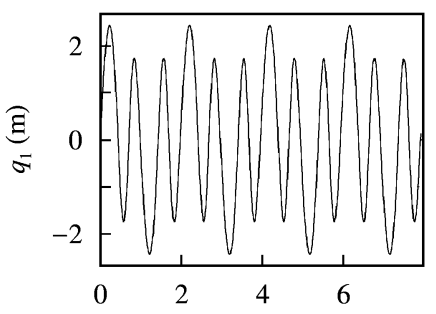

(a)

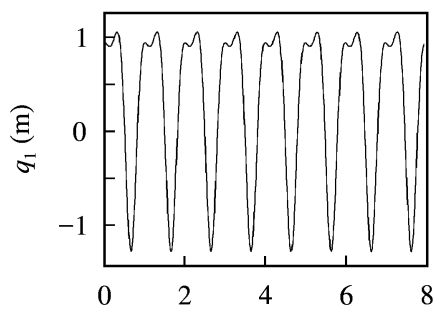

(b)
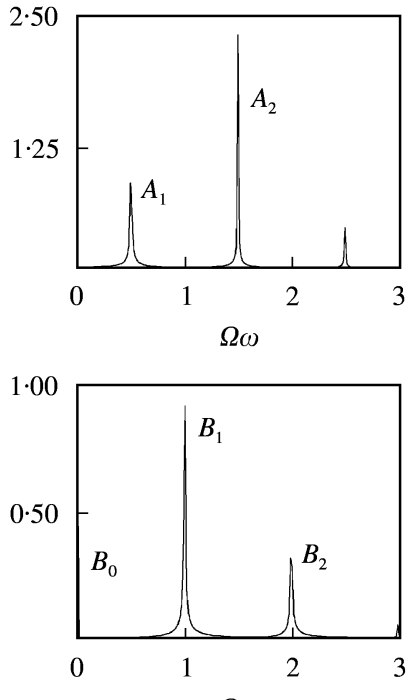

$\Omega \omega$

Figure 3. Coupled combinatorial harmonic response of the order $(n, m)=(3,2)$ : (a) time series and Fourier transform of $q_{1}(t)$; (b) time series and Fourier transform of $q_{2}(t) . \omega=2 \cdot 1 / \mathrm{s}$.
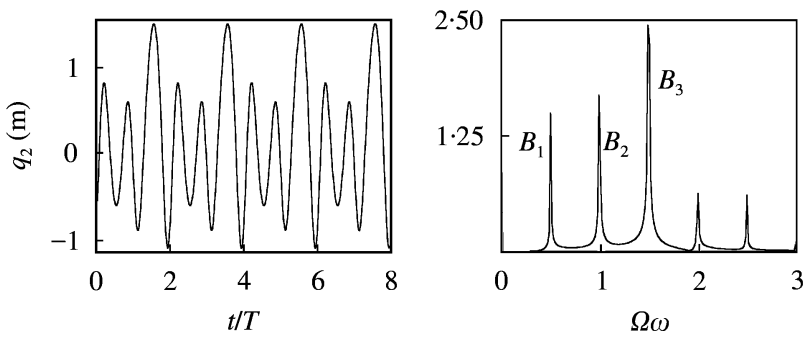

Figure 4. In-plane combinatorial harmonic response of the order $(n, m)=(3,2)$. Time series and Fourier transform of $q_{2}(t) . \omega=2 \cdot 15 / \mathrm{s}, U=0.5 \mathrm{~m}$.

corresponding results under identical cable parameters and initial conditions, when the circular excitation frequency has been been increased to $\omega=2 \cdot 15 / \mathrm{s}$. The coupled in-plane and out-of-plane components response becomes unstable and only the in-plane response persists irrespective of the initial conditions on $q_{1}(t)$. The circular eigenfrequency of the in-plane mode, $\omega_{2}=3 \cdot 2007 / \mathrm{s}$, is close to $\frac{3}{2} \omega$. The resulting in-plane response is dominated by a subharmonic component with the circular frequency $\frac{1}{2} \omega$ and a combinatorial harmonic component with the frequency $\frac{3}{2} \omega$, in addition to the harmonic component and the static drift. Both of the indicated periodic motions in the vicinity of $\frac{2}{3} \omega_{1}$ co-exist with an in-plane low-amplitude almost harmonical motion, attracting initial values in the vicinity of $q_{1}(0)=q_{2}(0)=\dot{q}_{1}(0)=\dot{q}_{2}(0)=0$.

From the indicated observations, the coupled in-plane and out-of-plane combinatorial harmonic response of the order $(n, m)=(3,2)$ is assumed to have the following form:

$$
\begin{aligned}
& q_{1}(t)=A_{1}(t) \cos \left(\frac{1}{2} \omega t+\Phi_{1}(t)\right)+A_{2}(t) \cos \left(\frac{3}{2} \omega t+\Phi_{2}(t)\right), \\
& \mathrm{q}_{2}(t)=B_{0}(t)+B_{1}(t) \cos \left(\omega t+\Psi_{1}(t)\right)+B_{2}(t) \cos \left(2 \omega t+\Psi_{2}(t)\right) .
\end{aligned}
$$


The amplitudes $A_{1}(t), A_{2}(t), B_{1}(t), B_{2}(t)$, the static drift $B_{0}(t)$, and the phases $\Phi_{1}(t), \Phi_{2}(t)$, $\Psi_{1}(t), \Psi_{2}(t)$ are assumed to be slowly varying functions with a large time scale compared to the period $2 T=2 \pi / \omega$ of the assumed motion. These quantities may then be assumed to be constant during the period $2 T$, and the differential equations for the long-term development may be derived by the averaging method. The singular points of these equations, representing possible combinatorial harmonical motions, become

$$
\begin{gathered}
-2 \zeta_{1} \omega_{1} \frac{1}{2} \omega A_{1}+\beta_{1}\left(A_{1} \sin 2 \Phi_{1}+A_{2} \sin \left(\Phi_{1}-\Phi_{2}\right)\right)=0 \\
\left(\omega_{1}^{2}-\frac{1}{4} \omega^{2}\right) A_{1}+\beta_{1}\left(A_{1} \cos 2 \Phi_{1}+A_{2} \cos \left(\Phi_{1}-\Phi_{2}\right)\right) \\
+\gamma_{1} A_{1}\left(A_{1}^{2}+2 A_{2}^{2}\right)+\gamma_{2} A_{1}\left(4 B_{0}^{2}+2 B_{1}^{2}+2 B_{2}^{2}\right)=0 \\
-2 \zeta_{1} \omega_{1} \frac{3}{2} \omega A_{2}-\beta_{1} A_{1} \sin \left(\Phi_{1}-\Phi_{2}\right)=0 \\
\left(\omega_{1}^{2}-\frac{9}{4} \omega^{2}\right) A_{2}+\beta_{1} A_{1} \cos \left(\Phi_{1}-\Phi_{2}\right) \\
+\gamma_{1} A_{2}\left(A_{2}^{2}+2 A_{1}^{2}\right)+\gamma_{2} A_{2}\left(4 B_{0}^{2}+2 B_{1}^{2}+2 B_{2}^{2}\right)=0 \\
\omega_{2}^{2} B_{0}+\beta_{2} B_{1} \cos \Psi_{1}+\gamma_{4} B_{0}\left(\frac{4}{3} B_{0}^{2}+2 B_{1}^{2}+2 B_{2}^{2}\right)+2 \gamma_{3} B_{0}\left(A_{1}^{2}+A_{2}^{2}\right)=0 \\
-2 \zeta_{2} \omega_{2} \omega B_{1}+\beta_{2}\left(2 B_{0} \sin \Psi_{1}+B_{2} \sin \left(\Psi_{1}-\Psi_{2}\right)\right)=F_{2} \sin \Psi_{1} \\
\left(\omega_{2}^{2}-\omega^{2}\right) B_{1}+\beta_{2}\left(2 B_{0} \cos \Psi_{1}+B_{2} \cos \left(\Psi_{1}-\Psi_{2}\right)\right) \\
+\gamma_{4} B_{1}\left(B_{1}^{2}+4 B_{0}^{2}+4 B_{2}^{2}\right)+2 \gamma_{3} B_{1}\left(A_{1}^{2}+A_{2}^{2}\right)=F_{2} \cos \Psi_{1} \\
-4 \zeta_{2} \omega_{2} \omega B_{2}-\beta_{2} B_{1} \sin \left(\Psi_{1}-\Psi_{2}\right)=0 \\
\left(\omega_{2}^{2}-4 \omega^{2}\right) B_{2}+\beta_{2} B_{1} \cos \left(\Psi_{1}-\Psi_{2}\right) \\
+\gamma_{4} B_{2}\left(B_{2}^{2}+4 B_{0}^{2}+2 B_{1}^{2}\right)+2 \gamma_{3} B_{2}\left(A_{1}^{2}+A_{2}^{2}\right)=0
\end{gathered}
$$

Various terms involving combinations of the amplitudes $A_{1}, A_{2}, B_{0}, B_{1}, B_{2}$ and the phases $\Phi_{1}, \Phi_{2}, \Psi_{1}$ and $\Psi_{2}$ have been omitted, either because the amplitudes or the sine or cosine functions of the phase combinations turn out to be insignificant compared to the retained terms. These approximations will be justified by later numerical validation of the model. As seen, combinatorial harmonic response of the order $(n, m)=(3,2)$ is caused by both the parametric excitation (unveiled by the parameters $\beta_{1}$ and $\beta_{2}$ ), as well as the external excitation, indicated by the amplitude $F_{2}$. The equations have to be solved by cyclic iteration. Initially, equation (62) is solved for $\sin \left(\Phi_{1}-\Phi_{2}\right)$ and $\cos \left(\Phi_{1}-\Phi_{2}\right)$ as follows:

$$
\begin{gathered}
\sin \left(\Phi_{1}-\Phi_{2}\right)=-3 \zeta_{1} \omega_{1} \omega \frac{A_{2}}{\beta_{1} A_{1}} \\
\cos \left(\Phi_{1}-\Phi_{2}\right)=-\left(\omega_{1}^{2}-\frac{9}{4} \omega^{2}+\gamma_{1} A_{2}^{2}+\gamma_{2}\left(4 B_{0}^{2}+2 B_{1}^{2}+2 B_{2}^{2}\right)\right) \frac{A_{2} \beta_{1}}{A_{1}}-2 \gamma_{1} \frac{A_{2}^{2}}{\beta_{1}^{2}} \frac{\beta_{1} A_{1}}{A_{2}},
\end{gathered}
$$

Equation (66) are squared and added, resulting in the following cubic equation in $A_{2}^{2}$ :

$$
a A_{2}^{2}+b A_{2}^{4}+c A_{2}^{6}=d,
$$


where

$$
\begin{aligned}
& a=9 \zeta_{1}^{2} \omega_{1}^{2} \omega^{2}+\left(\omega_{1}^{2}-\frac{9}{4} \omega^{2}+2 \gamma_{1} A_{1}^{2}+\gamma_{2}\left(4 B_{0}^{2}+2 B_{1}^{2}+2 B_{2}^{2}\right)\right)^{2}, \\
& b=2 \gamma_{1}\left(\omega_{1}^{2}-\frac{9}{4} \omega^{2}+2 \gamma_{1} A_{1}^{2}+\gamma_{2}\left(4 B_{0}^{2}+2 B_{1}^{2}+2 B_{2}^{2}\right)\right), \\
& c=\gamma_{1}^{2}, \quad d=\beta_{1}^{2} A_{1}^{2} .
\end{aligned}
$$

For the evaluation of $a, b, c$ and $d$ the values of $A_{1}, B_{0}, B_{1}, B_{2}$ from the previous iteration level are used. The cubic equation (67) only has a single positive real solution for $A_{2}^{2}$. This solution is applied in equation (66), which is used next in equation (62) to eliminate $\sin \left(\Phi_{1}-\Phi_{2}\right)$ and $\cos \left(\Phi_{1}-\Phi_{2}\right)$. The resulting equations are solved for $\cos 2 \Phi_{1}$ and $\sin 2 \Phi_{1}$ as follows:

$$
\begin{aligned}
\operatorname{Sin} 2 \Phi_{1}= & \frac{1}{\beta_{1}} \zeta_{1} \omega_{1} \omega+3 \beta_{1} \zeta_{1} \omega_{1} \omega X \\
\operatorname{Cos} 2 \Phi_{1}= & -\frac{1}{\beta_{1}}\left(\omega_{1}^{2}-\frac{1}{4} \omega^{2}+\gamma_{2}\left(4 B_{0}^{2}+2 B_{1}^{2}+2 B_{2}^{2}\right)\right) \\
& +\beta_{1}\left(\omega_{1}^{2}-\frac{9}{4} \omega^{2}+\gamma_{1} A_{2}^{2}+\gamma_{2}\left(4 B_{0}^{2}+2 B_{1}^{2}+2 B_{2}^{2}\right)\right) X-\frac{\gamma_{1}}{\beta_{1}^{3}} A_{2}^{2} \frac{1}{X},
\end{aligned}
$$

where $X=A_{2}^{2} / \beta_{1}^{2} A_{1}^{2}$. Squaring and summing equations (69) provides a quadratic equation in the quantity $X$, which has two positive and two negative real solutions. The positive real solutions determine two different solutions for the amplitude $A_{1}$. Each of these forms the basis for different solutions during the iteration procedure. Hence, two different combinatorial harmonic solutions to equations (61)-(65) exist. However, only one of these is stable, and can be realized by numerical integration of the modal co-ordinate equation.

From the observations in Figure 4, the in-plane combinatorial harmonic response of the order $(n, m)=(3,2)$ is assumed to have the following form:

$$
q_{2}(t)=B_{1}(t) \cos \left(\frac{1}{2} \omega t+\Psi_{1}(t)\right)+B_{2}(t) \cos \left(\omega t+\Psi_{2}(t)\right)+B_{3}(t) \cos \left(\frac{3}{2} \omega t+\Psi_{3}(t)\right) .
$$

Again, the amplitudes $B_{1}(t), B_{2}(t), B_{3}(t)$, and the phases $\Psi_{1}(t), \Psi_{2}(t), \Psi_{3}(t)$ are assumed to be slowly varying functions with a time scale which is large compared to the period $2 T=2 \pi / \omega$ of the assumed motion. Additionally, a static component, visible at the ordinate axis in Figure 4, as well as harmonic components with the circular frequencies $2 \omega$ and $2.5 \omega$ are present in the response. However, these components turn out to be less important and have been omitted in equation (70). Based on the averaging method the following equations may be derived for the singular points of the differential equations specifying the long-term development of these quantities:

$$
\begin{aligned}
& -2 \zeta_{2} \omega_{2} \frac{1}{2} \omega B_{1}+\beta_{2}\left(B_{1} \sin 2 \Psi_{1}+B_{3} \sin \left(\Psi_{1}-\Psi_{3}\right)\right)=0 \\
& \left(\omega_{2}^{2}-\frac{1}{4} \omega^{2}+\gamma_{4}\left(B_{1}^{2}+2 B_{2}^{2}+2 B_{3}^{2}\right)\right) B_{1}+\beta_{2}\left(B_{1} \cos 2 \Psi_{1}+B_{3} \cos \left(\Psi_{1}-\Psi_{3}\right)\right)=0 \\
& -\left(2 \zeta_{2} \omega_{2} \omega+2 \gamma_{4} B_{1} B_{3} \sin \left(\Psi_{1}-2 \Psi_{2}+\Psi_{3}\right)\right) B_{2}=F_{2} \sin \Psi_{2},
\end{aligned}
$$




$$
\begin{aligned}
& \left(\omega_{2}^{2}-\omega^{2}+\gamma_{4}\left(B_{2}^{2}+2 B_{1}^{2}+2 B_{3}^{2}\right)+2 \gamma_{4} B_{1} B_{3} \cos \left(\Psi_{1}-2 \Psi_{2}+\Psi_{3}\right)\right) B_{2}=F_{2} \cos \Psi_{2}, \\
& -2 \zeta_{2} \omega_{2} \frac{3}{2} \omega B_{3}-\beta_{2} B_{1} \sin \left(\Psi_{1}-\Psi_{3}\right)=0, \\
& \left(\omega_{2}^{2}-\frac{9}{4} \omega^{2}+\gamma_{4}\left(B_{3}^{2}+2 B_{1}^{2}+2 B_{2}^{2}\right)\right) B_{3}+\beta_{2} B_{1} \cos \left(\Psi_{1}-\Psi_{3}\right)=0 .
\end{aligned}
$$

As was the case for equations (61)-(65) various less important terms have been omitted in equations (71)-(73). The equations have to be solved by cyclic iteration in much the same way as for equations (61)-(65). Initially, equation (73) is solved for $\sin \left(\Psi_{1}-\Psi_{3}\right)$ and $\cos \left(\Psi_{1}-\Psi_{3}\right)$, and a cubic equation for $B_{3}^{2}$ is obtained upon squaring and adding these equations similar to equation (67), which turns out to have only a single positive solution for $B_{3}^{2}$. Next, $\sin \left(\Psi_{1}-\Psi_{3}\right)$ and $\cos \left(\Psi_{1}-\Psi_{3}\right)$ are eliminated from equation (71), and a quadratic equation may be formulated for $B_{1}^{2}$, which turns out to have two positive solutions. Each of these solutions generates two possible combinatorial harmonic solutions during the iteration procedure. Again only one of these turns out to be stable.

\section{NUMERICAL EXAMPLE}

The following data refer to the longest stay in the newly constructed cable stayed bridge across the Øresund between Denmark and Sweden.

$$
\begin{aligned}
& L_{c}=260 \mathrm{~m}, \quad A E=2.17 \times 10^{9} \mathrm{~N}, \quad F_{c}=5.5 \times 10^{6} \mathrm{~N}, \quad \mu_{0}=81.05 \mathrm{~kg} / \mathrm{m}, \\
& \zeta_{1}=\zeta_{2}=0.01, \quad U=0.15,0.5 \mathrm{~m}, \quad \theta=30.4^{\circ}, \quad g=9.81 \mathrm{~m} / \mathrm{s}^{2} .
\end{aligned}
$$

For this case

$$
\frac{f}{L_{c}}=4.0524 \times 10^{-3}, \quad \Omega_{2}=3.19460680 \Rightarrow \frac{\omega_{2}-\omega_{1}}{\omega_{1}}=1.687 \times 10^{-2} .
$$

The superharmonic response of the order $n=2$ for the modal amplitudes $A=Q_{1, n}$ and $B=Q_{2, n}$ has been shown in Figures 5 and 6 for the data in equation (74). The solid lines denote stable solutions, the dashed lines denote unstable solutions while the amplitude response results obtained from numerical integration of the differential equations (19)



Figure 5. Superharmonic response of the order $n=2 . U=0 \cdot 15 \mathrm{~m}:+$, numerical integration of equation (19), , stable solution. 

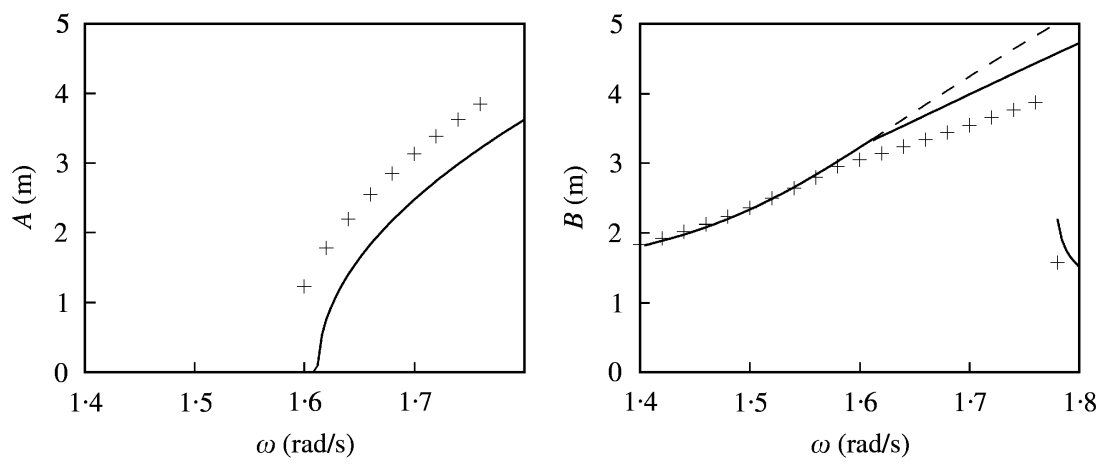

Figure 6. Superharmonic response of the order $n=2 . U=0.5 \mathrm{~m}:+$, numerical integration of equation (19), , stable solution, - - -, unstable in-plane solution.

are shown by + . Figure 5 shows that only in-plane motion exists for $U=0 \cdot 15 \mathrm{~m}$. Although the excitation is different, the results of Figure 5 are quite similar to those obtained in reference [19] for an in-plane superharmonic response due to in-plane harmonic excitation. Actually, the physical origin of the excitation is only felt through the modal load amplitude $P_{n}$ which in the present case is given by equation (45). For $U=0.5 \mathrm{~m}$, Figure 6 shows that only the in-plane motion exists as a stable solution below a bifurcation point where a whirling motion takes place. In a small frequency range above the bifurcation point, a stable in-plane superharmonic component co-exists with the whirling component (hardly visible in Figure 6(b)). However, the in-plane solution very soon loses stability, so in an interval up to approximately $\omega=1.78 \mathrm{rad} / \mathrm{s}$ the whirling motion is the only stable one. For a still larger excitation frequency a new stable in-plane component emerges at vibration levels below that of the whirling component which remains stable. The indicated theoretical stability properties of the analytical solutions are confirmed by the results obtained by numerical integration. For $U=0.5 \mathrm{~m}$ trajectories for the modal co-ordinates $q_{1}$ and $q_{2}$ of the combined harmonic and superharmonic components as given by equation (36) have been shown in Figure 7 for the data in equation (74) and excitation frequency $\omega=1.65 \mathrm{rad} / \mathrm{s}$. These trajectories of the whirling motion show that equation (36) gives acceptable results compared with the numerical results. As seen in Figure 7, the shape of the trajectories are rather insensitive to the damping ratio $\zeta_{2}$, whereas a relatively large value of $\zeta_{1}$ implies a marked change of the inclination of the curves.

Figure 8 shows the variation as a function of $\omega$ of the combinatorial amplitudes $A_{1}, A_{2}$, $B_{0}, B_{1}$ and $B_{2}$ obtained from equations (61)-(65) and equations (71)-(73) for $U=0 \cdot 5 \mathrm{~m}$. As mentioned, two possible combinatorial harmonic solutions to equations (61)-(65) exist, but only one of these is stable. The stable solution has been indicated by a solid line and the unstable solution by a dashed signature. It is seen that the out-of-plane amplitudes $A_{1}$ and $A_{2}$ evolve continuously without a discrete critical frequency below which $A_{1}=A_{2}$ (corresponding to purely in-plane motion). Correspondingly, the amplitudes $B_{0}, B_{1}$ and $B_{2}$ for the in-plane motion are not exactly identical below a certain frequency, although they converge to one another as the excitation frequency is reduced. In Figure 9, the stable and unstable solutions for $B_{1}, B_{2}$ and $B_{3}$ have been shown by a full line and dashed line respectively. Since purely harmonic response according to equation (70) implies that $B_{1}=B_{3}=0$, it is seen from the Figure that the in-plane combinatorial responses are bifurcating from the harmonic response at critical circular frequencies, in contrast to the whirling cases in Figure 8. In combination, Figures 8 and 9 show that in the vicinity of $\omega=2 \cdot 1 / \mathrm{s}$, both a stable in-plane and a coupled in-plane and out-of-plane combinatorial 

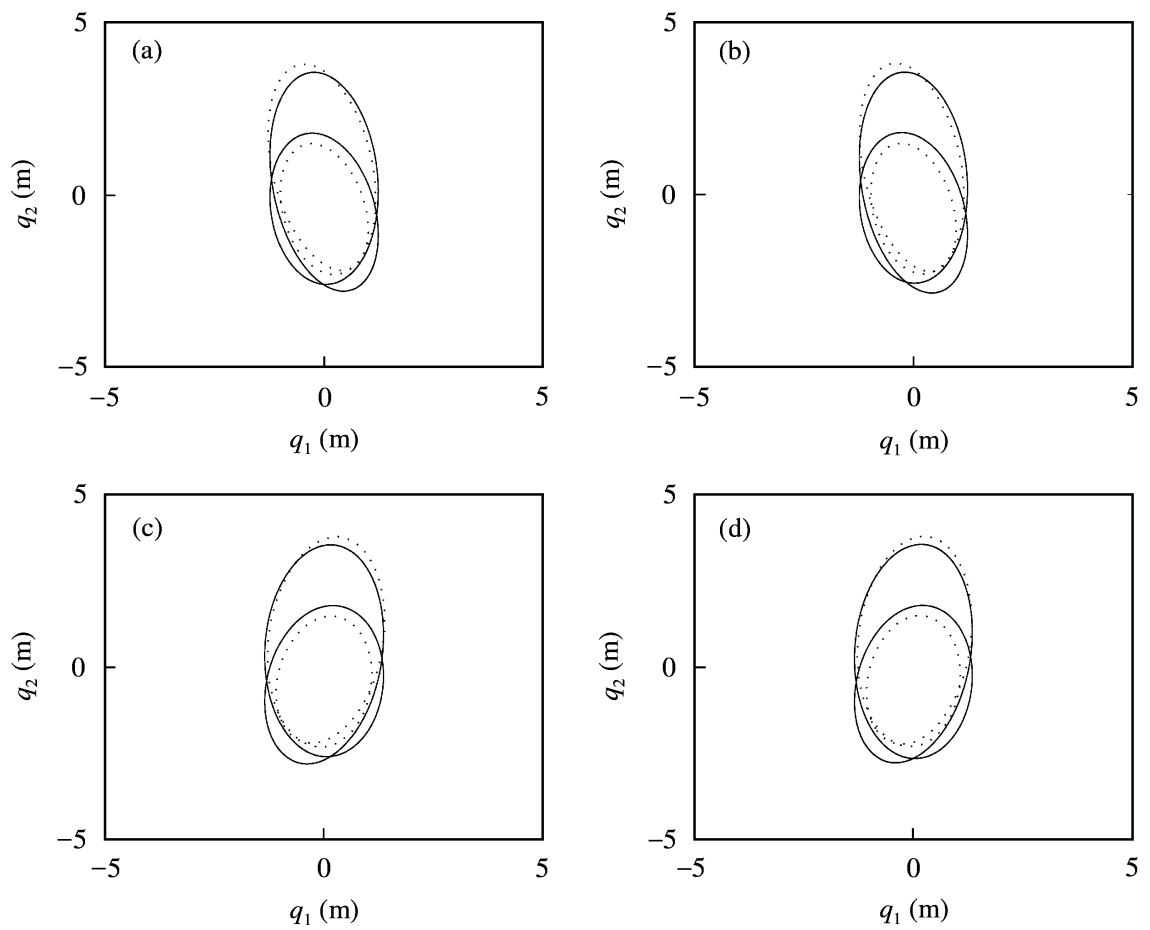

Figure 7. Trajectories of $q_{1}(t)$ and $q_{2}(t)$ in superharmonic whirling response. $U=0.5 \mathrm{~m}:---$, numerical integration of equation (19), $\stackrel{\longrightarrow}{-}$, stable analytical solution. (a) $\zeta_{1}=0 \cdot 01, \zeta_{2}=0 \cdot 01$; (b) $\zeta_{1}=0 \cdot 01, \zeta_{2}=0 \cdot 005$; (c) $\zeta_{1}=0 \cdot 005, \zeta_{2}=0 \cdot 01 ;$ (d) $\zeta_{1}=0 \cdot 005, \zeta_{2}=0 \cdot 005$.
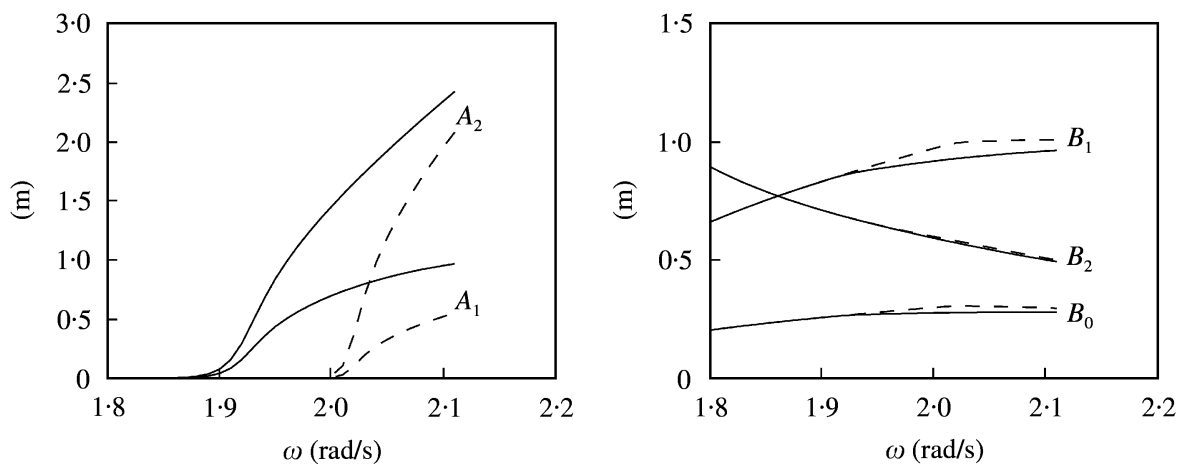

Figure 8. Amplitudes of coupled in-plane and out-of-plane combinatorial harmonic response of the order $(n, m)=(3,2) . U=0.5 \mathrm{~m}:-$, stable solution; - - - , unstable solution.

motion exists. Moreover, it is shown that one of these combinatorial harmonic responses of the order $(n, m)=(3,2)$ will dominate the cable response for $\omega \in[1 \cdot 9 / \mathrm{s}, 2 \cdot 2 / \mathrm{s}]$. Both the stable and unstable branches in Figures 8 and 9 disappear due to the appearance of complex solutions at sufficiently large circular frequencies. For the whirling motions this disappearance takes place for $\omega>2 \cdot 1 \mathrm{rad} / \mathrm{s}$ and for the combinatorial motion for $\omega>2 \cdot 2 \mathrm{rad} / \mathrm{s}$.

The coupled in-plane and out-of-plane combinatorial response trajectories for the modal co-ordinates $q_{1}$ and $q_{2}$ have been shown in Figure 10 as well as the excitation frequency $\omega=2 \cdot 1 \mathrm{rad} / \mathrm{s}$. These trajectories show that equation (60) gives acceptable results compared 


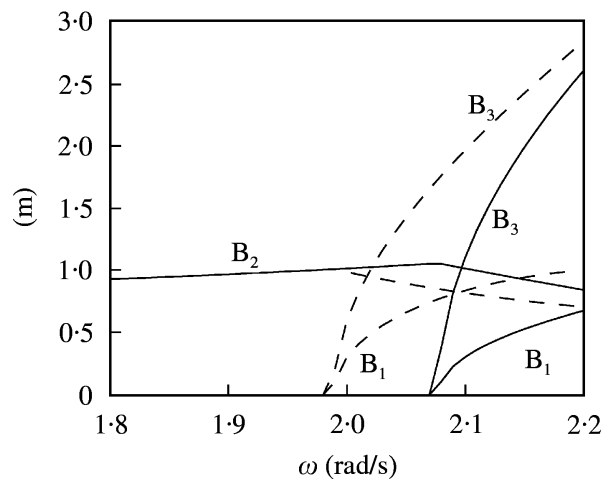

Figure 9. Amplitudes of in-plane combinatorial harmonic response of the order $(n, m)=(3,2) . U=0 \cdot 5 \mathrm{~m}:-$ stable solution; ----, unstable solution.
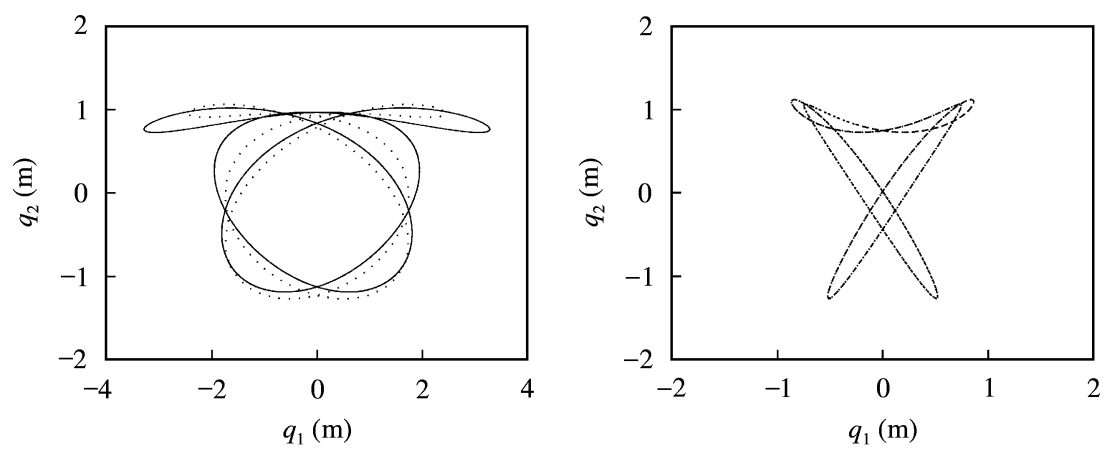

Figure 10. Trajectories of $q_{1}(t)$ and $q_{2}(t)$ for coupled in-plane and out-of-plane combinatorial harmonic whirling response of the order $(n, m)=(3,2) . U=0.5 \mathrm{~m}:---$, numerical integration of equation $(19) ;-\square$, analytical solution.

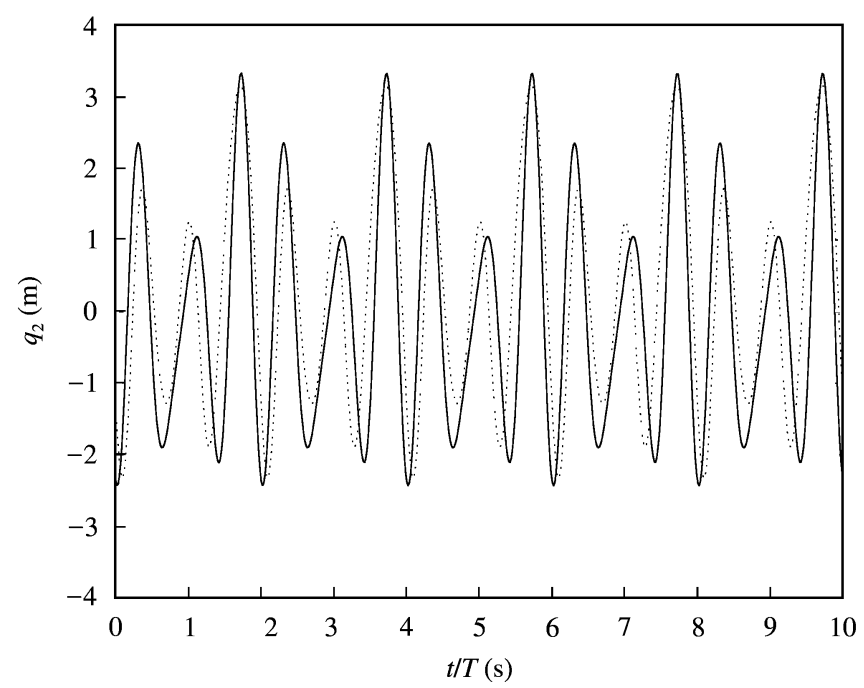

Figure 11. Time series of $q_{2}(t)$ for in-plane combinatorial harmonic response of the order $(n, m)=(3,2)$. $U=0.5 \mathrm{~m}:---$, numerical integration of equation (19), $\longrightarrow$, stable analytical solution.

with the numerical results. In Figure 10, both the stable and the unstable solutions are shown. The in-plane combinatorial response trajectory for the modal co-ordinates $q_{2}$ is shown in Figure 11 for frequency $\omega=2.15 \mathrm{rad} / \mathrm{s}$. Here it is also seen that the 
proposed model of equation (70) for the in-plane combinatorial response gives acceptable results.

\section{CONCLUSIONS}

The equations of motion for flexible linear elastic cables excited by forced support point motions have been derived assuming small axial strains. Assuming the sag-to-chord-length ratio to be small, the equations of motion are next discretized using the eigenmodes of the parabolic approximation to the static equilibrium suspension state as a functional basis. Since the emphasis is placed on the structural response due to harmonically varying support-point excitations with circular frequency well below the lowest eigenfrequencies of the cable, a two-degree-of-freedom model has been adopted, retaining only the lowest out-of-plane and the lowest in-plane mode in the modal expansion.

The superharmonic response and the combinatorial harmonic response have both been analyzed. In both cases, it is demonstrated that entirely in-plane response to the in-plane excitation may coexist with coupled in-plane and out-of-plane vibration. The coupled superharmonic response is developed continuously from the harmonic response without a marked bifurcation frequency. By contrast, such well-defined bifurcation points exist for the in-plane combinatorial response. All analytical results are based on the averaging method (first order Krylov-Bogoliubov-Mitropolsky perturbation method). The validity of the solutions obtained has been verified by direct numerical integration of the modal equations of motion. For this reason, good quantitative results are obtained at moderate amplitudes of the support point. However, acceptable agreement between numerical and analytical results was also obtained for a higher vibration level and for superharmonic response as well as combinatorial response.

\section{ACKNOWLEDGMENT}

The present work has been supported by The Danish Technical Research Council within the project "Damping Mechanisms in Dynamics of Structures and Materials".

\section{REFERENCES}

1. M. IRvine 1992 Cable Structures. New York: Dover.

2. M. Irvine and T. K. Caughey 1974 Royal Society of London, Series A 341, 299-315. The linear theory of free vibrations of a suspended cable.

3. P. HAGEDORN and B. SCHAFER 1980 International Journal of Nonlinear Mechanics 15, 333-339. On nonlinear free vibrations of an elastic cable.

4. A. Luongo, G. Rega and F. Vestroni 1984 International Journal of Nonlinear Mechanics 19, 39-52. Planar nonlinear vibrations of an elastic cable.

5. A. Luongo, G. Rega and F. Vestroni 1982 Journal of Sound and Vibration 82, 247-259. Monofrequency oscillations of a nonlinear model of a suspended cable.

6. G. REgA, F. VESTRONI and F. BENEDETTINI 1984 International Journal of Solids and Structures 20, 95-103. Parametric analysis of large amplitude free vibrations of a suspended cable.

7. F. Benedettini, G. RegA and F. Vestroni 1986 Meccanica 21, 38-46. Modal coupling in the free nonplanar finite motion of an elastic cable.

8. F. Benedettini and G. Rega 1987 International Journal of Nonlinear Mechanics 22, 497-509. Nonlinear dynamics of an elastic cable under planar excitation.

9. S. I. AL-NOURY and S. A. ALI 1985 Journal of Sound and Vibration 101, 451-462. Large amplitude vibrations of parabolic cables. 
10. G. V. RAO and R. IYengar 1991 Journal of Sound and Vibration 149, 25-41. Internal resonance and nonlinear response of elastic cable under periodic excitation.

11. K. TAKahashi and Y. Konishi 1987 Journal of Sound and Vibration 118, 85-97. Nonlinear vibrations of cables in three dimensions. Part II: out-of-plane vibration under in-plane sinusoidally time varying loading.

12. N. C. PERKINS 1992 International Journal of Nonlinear Mechanics 27, 233-250. Modal interactions in the nonlinear response of elastic cables under parametric/external excitation.

13. C. L. LeE and N. C. PERKIns 1992 Nonlinear Dynamics 3, 465-490. Nonlinear oscillations of suspended cables containing two-to-one internal resonance.

14. C. L. LEE and N. C. PERKINS 1992 Proceedings of ASME Winter Annual Meeting AMD-14, 59-67. Three-dimensional oscillations of suspended cables involving simultaneous internal resonance.

15. F. Benedettini, G. Rega and R. Alaggio 1995 Journal of Sound and Vibration 182, 775-798. Nonlinear oscillations of a four-degree-of-freedom model of a suspended cable under multiple internal resonance conditions.

16. G. Rega, R. Alaggio and F. Benedettini 1997 Nonlinear Dynamics 14, 89-117. Experimental Investigation of the nonlinear response of a hanging cable. Part I: local analysis.

17. F. Benedettini and G. RegA 1997 Nonlinear Dynamics 14, 119-138. Experimental Investigation of the nonlinear response of a hanging cable. Part II: global analysis.

18. G. Rega, W. Lacarbonara, A. H. Nayfeh and C. M. Chin 1997 International Journal of Nonlinear Dynamics 34, 901-924. Multiple resonances in suspended cables: direct versus reduced-order models.

19. F. BenEDETtini and G. RegA 1989 Journal of Sound and Vibration 132, 353-366. Planar nonlinear oscillations of elastic cables under superharmonic resonance conditions.

20. L. MeIrovitch 1975 Elements of Vibration Analysis. New York: McGraw-Hill.

\section{APPENDIX A}

Based on the parabolic approximations to eigenmodes (27) the following results may be evaluated for the tensor components entering equation (23) for $\alpha=1,2$ :

$$
\begin{aligned}
& e_{j} e_{k} a_{j k}^{(\alpha)}=8 \frac{f}{L_{c}} \frac{A E}{\mu_{0} L_{c}} \begin{cases}2 e_{2} e_{3} a_{1}, & \alpha=1, \\
\left(e_{k} e_{k}+2 e_{2}^{2}\right) a_{2}, & \alpha=2,\end{cases} \\
& e_{j} \sum_{\beta=1}^{2} c_{j}^{(\alpha \beta)} q_{\beta}=\frac{A E}{\mu_{0} L_{c}^{2}} e_{1} \begin{cases}q_{1} c_{1}, & \alpha=1, \\
\mathrm{q}_{2} c_{2}, & \alpha=2,\end{cases} \\
& \sum_{\beta=1}^{2} \sum_{\gamma=1}^{2} d^{(\alpha \beta \gamma)} q_{\beta} q_{\gamma}=\frac{f}{L_{c}} \frac{A E}{\mu_{0} L_{c}^{3}} \begin{cases}q_{1} q_{2} d_{1}, & \alpha=1, \\
q_{1}^{2} d_{2}+q_{2}^{2} d_{3}, & \alpha=2,\end{cases} \\
& e_{j} e_{k} \sum_{\beta=1}^{2} C_{j k}^{(\alpha \beta)} q_{\beta}=\frac{A E}{\mu_{0} L_{c}^{2}} \begin{cases}\left(2 e_{3}^{2}+e_{k} e_{k}\right) C_{1} q_{1}+2 e_{2} e_{3} C_{2} q_{2}, & \alpha=1, \\
2 e_{2} e_{3} C_{3} q_{1}+\left(2 e_{2}^{2}+e_{k} e_{k}\right) C_{4} q_{2}, & \alpha=2,\end{cases} \\
& e_{j} \sum_{\beta=1}^{2} \sum_{\gamma=1}^{2} D_{j}^{(\alpha \beta \gamma)} q_{\beta} q_{\gamma}=0 \text {, } \\
& \sum_{\beta=1}^{2} \sum_{\gamma=1}^{2} \sum_{\delta=1}^{2} G^{(\alpha \beta \gamma \delta)} q_{\beta} q_{\gamma} q_{\delta}=\frac{A E}{\mu_{0} L_{c}^{4}} \begin{cases}\left(G_{1} q_{1}^{2}+G_{2} q_{2}^{2}\right) q_{1}, & \alpha=1, \\
\left(G_{3} q_{1}^{2}+G_{4} q_{2}^{2}\right) q_{2}, & \alpha=2,\end{cases}
\end{aligned}
$$

where the introduced coefficients $a_{1}, a_{2}, c_{1}, c_{2}, d_{1}, d_{2}, d_{3}, C_{1}, C_{2}, C_{3}, C_{4}, G_{1}, G_{2}, G_{3}, G_{4}$ are functions of the non-dimensional frequency $\Omega_{2}$ defined as follows:

$a_{1}=\frac{2}{\pi}, \quad a_{2}=\frac{\left(2 \sin \Omega_{2} / 2-\Omega_{2} \cos \Omega_{2} / 2\right)\left(1-\cos \Omega_{2} / 2\right)}{2 \Omega_{2}+\Omega_{2} \cos \Omega_{2}-2 \sin \Omega_{2}}$, 


$$
\begin{aligned}
& c_{1}=\pi^{2}, \quad c_{2}=\frac{\Omega_{2}^{2}\left(\Omega_{2}-\sin \Omega_{2}\right)}{2 \Omega_{2}+\Omega_{2} \cos \Omega_{2}-2 \sin \Omega_{2}}, \\
& d_{1}=\frac{8 \pi^{2} \Omega_{2}}{1-\cos \Omega_{2} / 2}\left(2 \sin \frac{\Omega_{2}}{2}\left(\frac{1}{\Omega_{2}^{2}}+\frac{4 \pi^{2}+\Omega_{2}^{2}}{\left(4 \pi^{2}-\Omega_{2}^{2}\right)^{2}}\right)-\cos \frac{\Omega_{2}}{2}\left(\frac{1}{\Omega_{2}}-\frac{\Omega_{2}}{4 \pi^{2}-\Omega_{2}^{2}}\right)\right), \\
& d_{2}=\frac{4 \pi^{2} \Omega_{2}^{2}\left(1-\cos \Omega_{2} / 2\right)}{2 \Omega_{2}+\Omega_{2} \cos \Omega_{2}-2 \sin \Omega_{2}}\left(2 \sin \frac{\Omega_{2}}{2}\left(\frac{1}{\Omega_{2}^{2}}+\frac{4 \pi^{2}+\Omega_{2}^{2}}{\left(4 \pi^{2}-\Omega_{2}^{2}\right.}\right)^{2}\right) \\
& \left.-\cos \frac{\Omega_{2}}{2}\left(\frac{1}{\Omega_{2}}-\frac{\Omega_{2}}{4 \pi^{2}-\Omega_{2}^{2}}\right)\right), \\
& d_{3}=\frac{8 \Omega_{2}^{2}}{\left(1-\cos \Omega_{2} / 2\right)\left(2 \Omega_{2}+\Omega_{2} \cos \Omega_{2}-2 \sin \Omega_{2}\right)}\left(2 \sin \frac{\Omega_{2}}{2}\left(2+\frac{1}{3} \sin ^{2} \frac{\Omega_{2}}{2}\right)\right. \\
& \left.-\Omega_{2} \cos \frac{\Omega_{2}}{2}\left(3-\cos ^{2} \frac{\Omega_{2}}{2}\right)\right), \\
& \mathrm{C}_{1}=\frac{\pi^{2}}{2}, \quad C_{2}=-2 \frac{\Omega_{2} \pi^{2}}{1-\cos \Omega_{2} / 2} \frac{\cos \Omega_{2} / 2}{\Omega_{2}^{2}-\pi^{2}}, \\
& C_{3}=-2 \frac{\Omega_{2}^{2} \pi^{2}\left(1-\cos \Omega_{2} / 2\right)}{2 \Omega_{2}+\Omega_{2} \cos \Omega_{2}-2 \sin \Omega_{2}} \frac{\cos \Omega_{2} / 2}{\Omega_{2}^{2}-\pi^{2}}, \\
& C_{4}=\frac{\Omega_{2}^{2}}{2} \frac{\Omega_{2}-\sin \Omega_{2}}{2 \Omega_{2}+\Omega_{2} \cos \Omega_{2}-2 \sin \Omega_{2}}, \\
& G_{1}=\frac{3}{8} \pi^{4}, \quad G_{3}=\frac{\pi^{2}}{4} \frac{\Omega_{2}^{3}}{2 \Omega_{2}+\Omega_{2} \cos \Omega_{2}-2 \sin \Omega_{2}}\left(1-\frac{\left(2 \Omega_{2}^{2}-\pi^{2}\right) \sin \Omega_{2}}{\left(\Omega_{2}^{2}-\pi^{2}\right) \Omega_{2}}\right), \\
& G_{2}=\frac{\pi^{2}}{4} \frac{\Omega_{2}^{2}}{\left(1-\cos \Omega_{2} / 2\right)^{2}}\left(1-\frac{\left(2 \Omega_{2}^{2}-\pi^{2}\right) \sin \Omega_{2}}{\left(\Omega_{2}^{2}-\pi^{2}\right) \Omega_{2}}\right) \\
& G_{4}=\frac{1}{16} \frac{\Omega_{2}^{4}}{\left(1-\cos \Omega_{2} / 2\right)^{2}} \frac{6 \Omega_{2}+\sin \Omega_{2}-8 \sin \Omega_{2}}{2 \Omega_{2}+\Omega_{2} \cos \Omega_{2}-2 \sin \Omega_{2}} .
\end{aligned}
$$

